\title{
A Variational Model for Object Segmentation Using Boundary Information and Shape Prior Driven by the Mumford-Shah Functional
}

\author{
XAVIER BRESSON, PIERRE VANDERGHEYNST AND JEAN-PHILIPPE THIRAN \\ Signal Processing Institute, Swiss Federal Institute of Technology, EPFL-STI-ITS-Station 11, \\ CH-1015 Lausanne, Switzerland \\ Xavier.Bresson@epfl.ch \\ Pierre.Vandergheynst@epfl.ch \\ JP.Thiran@epfl.ch
}

Received May 14, 2004; Revised October 25, 2005; Accepted November 16, 2005

First online version published in March, 2006

\begin{abstract}
In this paper, we propose a new variational model to segment an object belonging to a given shape space using the active contour method, a geometric shape prior and the Mumford-Shah functional. The core of our model is an energy functional composed by three complementary terms. The first one is based on a shape model which constrains the active contour to get a shape of interest. The second term detects object boundaries from image gradients. And the third term drives globally the shape prior and the active contour towards a homogeneous intensity region. The segmentation of the object of interest is given by the minimum of our energy functional. This minimum is computed with the calculus of variations and the gradient descent method that provide a system of evolution equations solved with the well-known level set method. We also prove the existence of this minimum in the space of functions with bounded variation. Applications of the proposed model are presented on synthetic and medical images.
\end{abstract}

Keywords: image segmentation, variational model, active contour, level set method, shape prior, shape registration, principal components analysis, Mumford-Shah model

\section{Introduction and Motivations}

This work aims at proposing a method to segment structures of interest whose global shape is given. The segmentation problem remains fundamental in the computer vision and image processing fields since it is a core component towards e.g. automated vision systems and medical applications.

Various methods have been proposed to extract objects of interest in images such as (Kass et al., 1987; Cootes and Taylor, 1999; Cremers et al., 2002). However these methods utilize parametric shape representations whose use is not as convenient as intrinsic representations such as the level set function
(Osher and Sethian, 1988). Other models such as geodesic/geometric active contours (Caselles et al., 1997; Kichenassamy et al., 1996) use this intrinsic modeling of contours e.g. to detect fine real-world shapes such as medical structures (Malladi et al., 1996; Yezzi et al., 1997; Jonasson et al., 2005). Level setbased methods are of growing interest since they are independent of the contour parametrization, and thus enable dealing with topological changes. They also benefit from efficient numerical schemes and are naturally extensible to higher dimensions. Despite of these great advantages, the first-generation active contours, based on image gradients, are highly sensitive to the presence of noise and poor image contrast, which 
can lead to bad segmentation results. To overcome this drawback, some authors have incorporated robust region-based evolution criteria into active contour energy functionals built from intensity statistics and homogeneity requirements (Paragios and Deriche, 2002; Chan and Vese, 2001; Jehan-Besson et al., 2003). Yet the segmentation of structures of interest with these second-generation active contours is not able to deal with occlusion problems or presence of strongly cluttered background. Therefore the integration of prior shape knowledge about the objects in the segmentation task represents a natural way to solve occlusion problems (Leventon et al., 2000; Paragios et al., 2003; Chen et al., 2002) and can be considered as a third generation of active contours. As we will see, we propose a model that exploits the advantages of the three generations of active contours.

The shape prior can be defined by different models such as Fourier descriptors, medial axis or atlas-based parametric models. Recently, the level set representation of shapes has been employed as a shape model (Leventon et al., 2000; Paragios et al., 2003; Charpiat et al., 2003). This shape description presents strong advantages since parametrization free, it can represent shapes of any dimension such as curves, surfaces and hyper-surfaces and basic geometric properties such as the curvature and the normal to contours are easily deduced. Finally, this shape representation is also naturally consistent with the level set framework of active contours. In Leventon et al. (2000), authors have used a level set representation to model the shape prior. They have defined a shape model of the object of interest by computing a principal components analysis (PCA) of training shapes embedded in level set functions. They have then integrated this shape model in an evolution equation to globally drive the active contour towards the prior shape. However, their evolution equation is not expressed by a partial differential equation (PDE) and there is no variational formulation associated with his evolution equation. In a general way, a variational formulation is useful both to understand and justify the proposed method. Moreover, evolution equations naturally appear from the variational model through the minimization of the proposed energy functional thanks to the calculus of variations. Finally a variational formulation of a problem helps in proving mathematical existence of solutions. Thus we propose a variational approach following the energy functional model of Chen et al. (2002) where we integrate the shape prior of Leventon et al. We then add a region-based energy term based on the Mumford-Shah functional (Mumford and Shah, 1989) to improve the robustness of our segmentation model with respect to (w.r.t.) noise, poor image contrast and initial position of the contour as shown in Paragios and Deriche (2002), Chan and Vese (2001), and Jehan-Besson et al. (2003). We will also prove the existence of a solution for our variational segmentation problem.

The organization of the paper is as follows. In Section 2, we briefly review some state-of-the-art methods which are directly related to our work. In Section 3, we define our variational model to address the object segmentation problem with a prior shape knowledge and we derive the system of evolution equations minimizing the proposed energy. We present in Section 4 results of the proposed method on 2-D synthetic and medical images. We discuss our segmentation model and compare it with other ones in Section 5 and conclude in Section 6. Finally, we prove in appendix the existence of a minimizer for our variational segmentation model.

\section{Shape-Based Active Contours and PCA Shape Modeling}

\subsection{Shape-Based Active Contours}

In this section, we review the main active contour models that use a shape prior.

In Leventon et al. (2000) and Leventon (2000), authors have developed active contours that use a shape model defined by a PCA. In their approach, the active contour evolves locally based on image gradients and curvature and globally towards the maximum a posteriori (MAP) probability of position and shape of the prior shape model. However, this a posteriori probability is maximized at each iteration by an independent optimization process, which means that the final evolution equation is not a PDE since two independent stages are necessary to evolve the surface. The evolution equation is the following:

$$
\begin{aligned}
u(t+1)= & u(t)+\lambda_{1}(g(c+\kappa)|\nabla u(t)| \\
& +\langle\nabla u(t), \nabla g\rangle)+\lambda_{2}\left(u^{\star}(t)-u(t)\right),(1)
\end{aligned}
$$

where $u^{\star}$ is the shape prior. The second term of the right-hand side of (1) weighted by $\lambda_{1}$ represents the classical term of the geodesic active contour. And the third term depending on $\lambda_{2}$ drives the shape of the active contour towards the shape prior given by the MAP estimation. 
Tsai et al. (2001, 2003), have integrated the shape model of Leventon et al. into the reduced version of the Mumford-Shah functional proposed by Chan and Vese (2001) and into two other segmentation models proposed by Yezzi et al. (1999) to segment images containing known object types. The implicit shape prior $\Phi[\mathbf{w}, \mathbf{p}]$, which depends on a shape vector $\mathbf{w}$ and a pose vector $\mathbf{p}$, is introduced into three energy functionals for the object segmentation such as the one based on the Chan and Vese model (Chan and Vese, 2001):

$$
E(\mathbf{w}, \mathbf{p})=-\left(\frac{S_{u}^{2}}{A_{u}}+\frac{S_{v}^{2}}{A_{v}}\right) .
$$

where $A_{u}=\int_{\Omega} H(-\Phi[\mathbf{w}, \mathbf{p}]), S_{u}=\int_{\Omega} I H(-\Phi$ $[\mathbf{w}, \mathbf{p}])$ are the area and the sum intensity $(I$ is the given image) in the region $R^{u}=\left\{(x, y) \in \mathbf{R}^{2}: \Phi(x, y)<0\right\}$ and $A_{v}=\int_{\Omega} H(\Phi[\mathbf{w}, \mathbf{p}]), S_{v}=\int_{\Omega} I H(\Phi[\mathbf{w}, \mathbf{p}])$ are the area and the sum intensity in the region $R^{v}=\left\{(x, y) \in \mathbf{R}^{2}: \Phi(x, y)>0\right\}$. The parameters $\mathbf{w}$ and $\mathbf{p}$ that optimize the segmentation energy functional (2) are given by two gradient descents.

Chen et al. (2002) have designed a novel variational model that incorporates prior shape knowledge into geometric/geodesic active contours. On the contrary to Leventon's approach, the shape model $C^{\star}$ of Chen is not a probabilistic one. It is computed as the average of a training set of rigidly aligned curves. However, this variational approach proves the existence of a solution minimizing their energy functional, which is not the case in the Leventon's method. Chen's functional is defined as:

$$
\begin{aligned}
F^{C}(C, \mu, \theta, T)= & \int_{0}^{1}(g(|\nabla I(C(p))|) \\
& \left.+\frac{\lambda}{2} d^{2}(\mu R C(p)+T)\right)\left|C^{\prime}(p)\right| d p
\end{aligned}
$$

where $C$ is the active contour, $(\mu, \theta, T)$ are the parameters of a rigid transformation (scale, orientation and translation) and $d$ is the distance to $C^{\star}$, the target shape. This functional is thus minimized when the active contour has captured both high image gradients and the shape prior. They have showed the good ability of the model to extract real-world structures in which the complete boundary was either missing or had low resolution and contrast (Chen et al., 2002).

Paragios et al. (2003) have built a new level set representation of shape from a training set in order to capture both global and local shape variations. They have used it to non-rigidly register two shapes and to segment objects with a modified version of the geodesic active regions defined in Paragios and Deriche (2002).

Cremers et al. (2002) have modified the MumfordShah functional to incorporate two statistical models of parametric shape in order to efficiently segment known objects in presence of misleading information due to noise, occlusion and strongly cluttered background. Concerning the shape model, they have used a multivariate Gaussian distribution in Cremers et al. (2002) and a nonlinear shape statistic derived from an extension of the kernel PCA in Cremers et al. (2002b). Then, Cremers et al. proposed a statistical shape prior, based on kernel density estimation, for level set based Mumford-Shah segmentation.

Let us finally mention the paper of Riklin-Raviv et al. (2004) which addresses the problem of general perspective transformations between a prior shape and an image to be segmented in the level set framework.

\subsection{The Shape Model of Leventon et al.}

2.2.1. Definition. This shape model is based on the PCA that aims at capturing the main variations of a training set while removing redundant information. Cootes and Taylor (1999) have used this technique on parametric contours to segment different kind of objects. The new idea introduced by Leventon et al. (2000) is to apply the PCA not on the parametric geometric contours but on the signed distance functions (SDFs) of these contours which are implicit and parameter free representations. They justified this choice in two ways. Firstly, SDFs provide a stronger tolerance than the parametric curves to slight misalignments during the alignment process of the training data since the values of neighboring pixels are highly correlated in a SDF. Secondly, this intrinsic contour representation also improves the shape registration process in terms of robustness, accuracy and speed. Indeed, the problem of the point-wise correspondence of contours (landmarks correspondence) is replaced by a problem of intensity correspondence on grid points which is easier to solve.

From a geometric point of view, the PCA analysis determines the best orthonormal basis $\left\{\mathbf{e}_{1} \ldots \mathbf{e}_{m}\right\}$ of $\mathbf{R}^{m}$ to represent a set of $n$ points $\left\{\phi_{1} \ldots \phi_{n}\right\}$ in the sense of the least squares fitting. Vectors $\left\{\mathbf{e}_{i}\right\}$ are given by the eigenvectors of the covariance matrix $\Sigma=\frac{1}{n} M M^{\top}$ where $M$ is a matrix whose column vectors are the $n$ aligned training SDFs $\left\{\phi_{j}\right\}$. Vectors $\left\{\mathbf{e}_{i}\right\}$ correspond to the 
principal variation directions of the set of $n$ points. They are called the principal components. Moreover, the first $p$ principal axes define a reduced $p$-dimensional vector space in $\mathbf{R}^{m}$ equivalent to a hyper-plane minimizing the sum of squared distances between this hyper-plane and the set of $n$ points. It is important to note that the accuracy of the fitting of this $p$-D hyper-plane in relation to the set of points can be measured in percentage by the formula $\beta=\sum_{k=1}^{p} \lambda_{k} / \sum_{k=1}^{n} \lambda_{k}$ where $\lambda_{k}$ are the eigenvalues of $\Sigma$. Thus, it is possible to arbitrarily fix the fitting percentage $\beta$ and represent the data in a sub-vector space of dimension $p$. In practice, only the first principal modes are necessary to model the biggest variations present in our training set. These $p$ principal components are sorted in a matrix $\mathbf{W}_{p}$.

Finally, the PCA can produce a new data based on the training set $\left\{\phi_{j}\right\}$ :

$$
\hat{\phi}=\bar{\phi}+\mathbf{W}_{p} \mathbf{x}_{\mathrm{pca}}
$$

where $\mathbf{x}_{\mathrm{pca}}$ is called the vector of eigencoefficients, the shape vector or the eigenmodes of variation.

2.2.2. Examples. In this paper, we have considered two sets of 2-D shapes of interest: one containing ellipses and the other one left brain ventricles. For the ellipse, we have generated a training set of 30 ellipses by changing the size of a principal axis with a Gaussian probability function and applied the PCA on the SDFs of 30 training ellipses. We have obtained one principal component that fits at $98 \%$ the set of ellipses. Figure 1 shows the aligned training ellipses and the shape function corresponding to the mean and the eigenmode of variation of the training set.

For the left brain ventricle, we have employed 2-D medical images. We have extracted 45 2-D images of left ventricles from several coronal slices of T1-Weighted Magnetic Resonance images (MRI) of healthy voluntaries (Fig. 2) to build our shape model. We have applied the PCA and obtained three principal components that fit at $88.2 \%$ the set of 45 SDFs of ventricles. Figure 3 shows the aligned training ventricles and the shape function corresponding to the mean and the three main eigenmodes of variation of the training set.

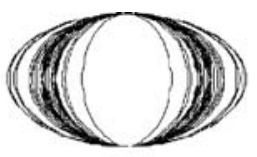

(a)

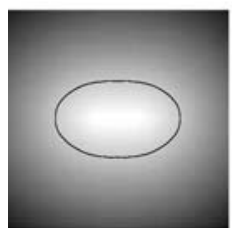

(b) $-2 \lambda_{1}$

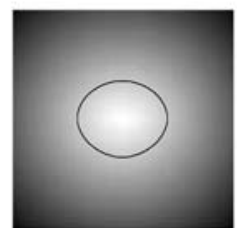

(c) Mean (d) $2 \lambda_{1}$

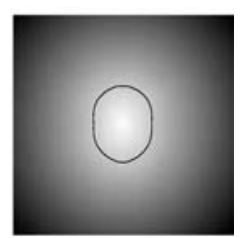

Figure 1. Figure (a) presents the 30 aligned training ellipses with the mean ellipse in dotted line. Figure (c) shows the mean value $\bar{\phi}$. Figures (b) and (d) present $\bar{\phi} \pm 2 \lambda_{1} \mathbf{e}_{1}$, the unique eigenmode of variation of SDF ellipses whose $\lambda_{1}$ is the eigenvalue. The zero level sets of the shape function $\hat{\phi}$ is plotted in solid dark line.
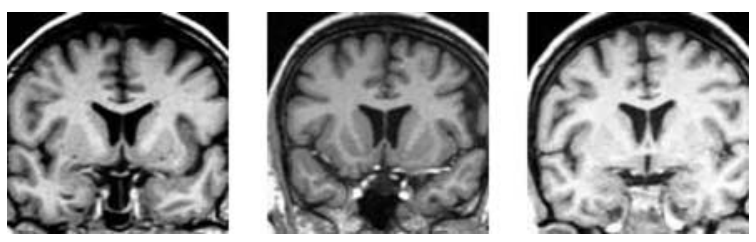

Figure 2. Three T1-Weighted Magnetic Resonance images of brain.

\section{Our New Object Segmentation Model}

\subsection{The Proposed Energy Functional}

We propose the following energy functional to address the problem of object segmentation using a geometric shape prior with local and global image information:

$$
\begin{aligned}
F= & \beta_{s} F_{\text {shape }}\left(C, \mathbf{x}_{\mathrm{pca}}, \mathbf{x}_{T}\right)+\beta_{b} F_{\text {boundary }}(C) \\
& +\beta_{r} F_{\text {region }}\left(\mathbf{x}_{\mathrm{pca}}, \mathbf{x}_{T}, u_{\mathrm{in}}, u_{\text {out }}\right)
\end{aligned}
$$

with

$$
\begin{aligned}
F_{\text {shape }} & =\oint_{0}^{1} \hat{\phi}^{2}\left(\mathbf{x}_{\mathrm{pca}}, h_{\mathbf{x}_{T}}(C(q))\right)\left|C^{\prime}(q)\right| d q \\
F_{\text {boundary }} & =\oint_{0}^{1} g(|\nabla I(C(q))|)\left|C^{\prime}(q)\right| d q \\
F_{\text {region }} & =\int_{\Omega_{\mathrm{in}}\left(\mathbf{x}_{\mathrm{pca}}, \mathbf{x}_{T}\right)}\left(\left|I-u_{\mathrm{in}}\right|^{2}+\mu\left|\nabla u_{\mathrm{in}}\right|^{2}\right) d \Omega \\
& +\int_{\Omega_{\mathrm{out}}\left(\mathbf{x}_{\mathrm{pca}}, \mathbf{x}_{T}\right)}\left(\left|I-u_{\mathrm{out}}\right|^{2}+\mu\left|\nabla u_{\mathrm{out}}\right|^{2}\right) d \Omega,
\end{aligned}
$$




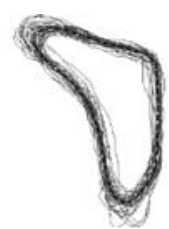

(a)
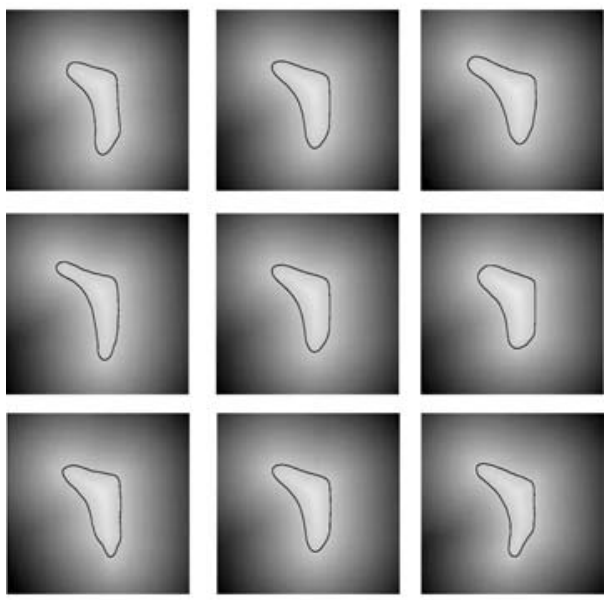

(i)

Figure 3. Figure (a) presents the 45 aligned training ventricles with the mean left ventricle in dotted line. On Figure (i), the middle column is the mean value and each row presents an eigenmode of variation of ventricles. The zero level sets of the shape function $\hat{\phi}$ is plotted in solid dark line.

where $C$ is the active contour, $\hat{\phi}$ is the shape function of the object of interest given by the PCA (see Eq. (4)), $\mathbf{x}_{\mathrm{pca}}$ is the vector of PCA eigencoefficients, $h_{\mathbf{x}_{T}}$ is an element of a group of geometric transformations parametrized by $\mathbf{x}_{T}$ (the vector of parameters), $g$ is an edge detecting function, $\Omega_{\text {in }}$ and $\Omega_{\text {out }}$ are the inside and outside regions of the zero level set of $\hat{\phi}$, $u_{\text {in }}$ and $u_{\text {out }}$ are smooth approximations of the original image $I$ in $\Omega_{\text {in }}$ and $\Omega_{\text {out }}$ and $\beta_{b}, \beta_{s}, \beta_{r}$ are arbitrary positive constants that balance the contributions of the boundary, shape and region terms.

The proposed functional $F$ is an extension of the work of Chen et al. (2002) where we have integrated the shape model of Leventon et al. (2000) and the Mumford-Shah functional (Mumford and Shah, 1989; Vese and Chan, 2002). In the following sections, we will analyze the shape and region terms.

\subsection{The Shape Term $F_{\text {shape }}$}

$F_{\text {shape }}$ is a functional introduced by the authors in Bresson et al. (2003) that depends on the active con-

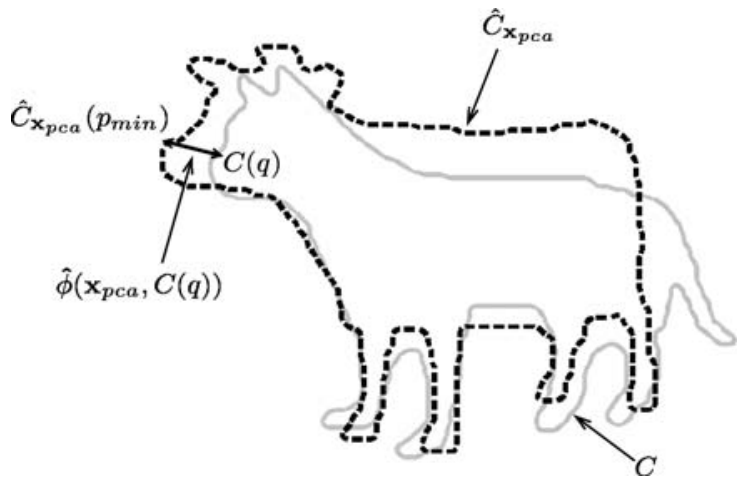

Figure 4. Illustration of the function $\hat{\phi}\left(\mathbf{x}_{\mathrm{pca}}, C(q)\right)$ : the square shape function is approximatively equal to the square Euclidean distance between the point $C(q)$ and the closest point $\hat{C}_{\mathbf{x}_{\mathrm{pca}}}\left(p_{\min }\right)$ on the zero level set $\hat{C}_{\mathbf{x}_{\mathrm{pca}}}$ of $\hat{\phi}\left(\mathbf{x}_{\mathrm{pca}}\right)$.

tour $C$, the vector $\mathbf{x}_{\mathrm{pca}}$ of PCA eigencoefficients and the vector $\mathbf{x}_{T}$ of geometric transformations. This functional evaluates the shape difference between the contour $C$ and the zero level set $\hat{C}$ of the shape function $\hat{\phi}$ provided by the PCA. It is an extension of the shapebased term of Chen et al. (2002) coupled with the shape model of Leventon et al. (2000). To give an interpretation of $F_{\text {shape }}$, let us take a rigid transformation with the scale parameter equal to one, the angle and the vector of translations equal to zero, Thus, the function $\hat{\phi}^{2}$ at the point $C(q)$ is:

$$
\begin{aligned}
\hat{\phi}^{2}\left(\mathbf{x}_{\mathrm{pca}}, h_{\mathbf{x}_{T}}(C(q))\right) & =\hat{\phi}^{2}\left(\mathbf{x}_{\mathrm{pca}}, C(q)\right) \\
& \simeq\left|\hat{C}_{\mathbf{x}_{\mathrm{pca}}}\left(p_{\mathrm{min}}\right)-C(q)\right|^{2}
\end{aligned}
$$

where $|\cdot|$ stands for the Euclidean norm.

The equality is not strict since the shape function $\hat{\phi}$ is not a SDF as Leventon noticed in Leventon et al. (2000) and Leventon (2000). However, the PCA applied on aligned SDFs of a training set produces shape functions very close to SDFs. The case of a strict equality in Eq. (9), i.e. the case of $\hat{\phi}$ is a true SDF, will be discussed in Section 5. Figure 4 illustrates the function $\hat{\phi}$ and $\hat{C}_{\mathbf{x}_{\mathrm{pca}}}\left(p_{\text {min }}\right)$. In practice, the point $\hat{C}_{\mathbf{x}_{\mathrm{pca}}}\left(p_{\mathrm{min}}\right)$ is not computed. It corresponds to the closest point of $C(q)$ on the zero level set of $\hat{\phi}$ and we used it to illustrate the shape function at point $C(q)$. Indeed, the shape function $\hat{\phi}^{2}\left(\mathbf{x}_{\mathrm{pca}}, h_{\mathbf{x}_{T}}(C(q))\right)$ is equal to the distance $\left|\hat{C}_{\mathbf{x}_{\mathrm{pca}}}\left(p_{\min }\right)-C(q)\right|^{2}$, i.e. the value of the level set-based function $\hat{\phi}$ at the point $C(q)$.

Finally, $F_{\text {shape }}$ is obtained by integrating $\hat{\phi}^{2}$ along the active contour, which defines the shape similarity 
measure equivalent to the sum of square differences (SSD). The minimization of $F_{\text {shape }}$ allows us to increase the similarity between the active contour and the shape model. The functional is minimized using the calculus of variations and the gradient descent method which provide three flows acting on the curve $C$, the vector of eigencoefficients $\mathbf{x}_{\text {pca }}$ and the vector of geometric transformations $\mathbf{x}_{T}$.

We analyze each of the three flows by fixing the two others. The flow minimizing $F_{\text {shape }}$ w.r.t. the curve $C$ is the classical geodesic flow (Caselles et al., 1997; Kichenassamy et al., 1996):

$$
\left\{\begin{aligned}
\partial_{t} C(t, q)= & \left(\hat{\phi}^{2} \kappa-\left\langle\nabla \hat{\phi}^{2}, \mathcal{N}\right\rangle\right) \mathcal{N} \\
& \text { in }] 0, \infty[\times[0,1] \\
C(0, q)= & C_{0}(q) \text { in }[0,1]
\end{aligned}\right.
$$

The first term of the right-hand side of the flow (10) is a mean curvature flow weighted by the square shape function $\hat{\phi}$ and the second term is a flow which pushes the contour $C$ toward the zero level set of $\hat{\phi}$, i.e. the contour $\hat{C}$, thanks to the vector field $\nabla \hat{\phi}^{2}$ which is close to a (square) distance function. The PDE defined in Eq. (10) changes the active contour shape into any shape provided by the PCA model. This shape morphing has two main advantages. First, it is independent of the contour parametrization because of the intrinsic level set representation. This means that the landmarks correspondence problem is replaced by a grid point-wise intensity correspondence which is easier to solve. Then, it is more accurate than parametrized shape morphing since the degree of deformation of level set functions is higher. Figure 5 presents the morphing between two curves.

The flow minimizing $F_{\text {shape }}$ w.r.t. the vector of eigencoefficients $\mathbf{x}_{\mathrm{pca}}$ is:

$$
\left\{\begin{array}{r}
d_{t} \mathbf{x}_{\mathrm{pca}}(t)=-2 \int_{0}^{1} \hat{\phi} \nabla_{\mathbf{x}_{\mathrm{pca}}} \hat{\phi}\left|C^{\prime}\right| d q \\
\text { in }] 0, \infty\left[\times \Omega_{\mathrm{pca}},\right. \\
\mathbf{x}_{\mathrm{pca}}(t=0)=\mathbf{x}_{\mathrm{pca}_{0}} \text { in } \Omega_{\mathrm{pca}} . \\
\text { with } \nabla_{\mathbf{x}_{\mathrm{pca}}} \hat{\phi}=\left(\begin{array}{l}
\mathbf{e}_{\mathrm{pca}}^{1} \\
\vdots \\
\mathbf{e}_{\mathrm{pca}}^{p}
\end{array}\right),
\end{array}\right.
$$

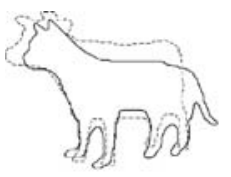

(a)

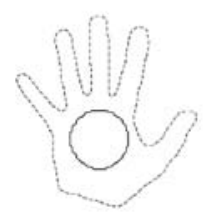

(d)

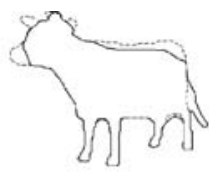

(b)

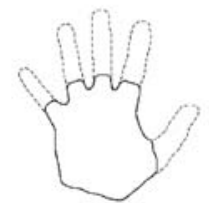

(e)

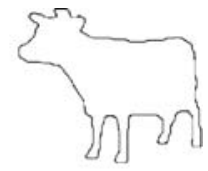

(c)

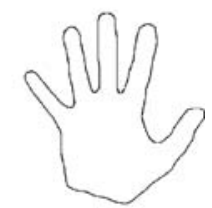

(f)
Figure 5. Minimization of $F_{\text {shape }}$ with the flow given in Eq. (19), $\mathbf{x}_{T}$ and $\mathbf{x}_{\text {pca }}$ being fixed. Active contour is in solid line and the shape prior in dotted line. Figures (a)-(c) show the matching of a cat (initial active contour) into a cow (shape prior). Figures (d)-(f) present the matching of a circle into a hand.
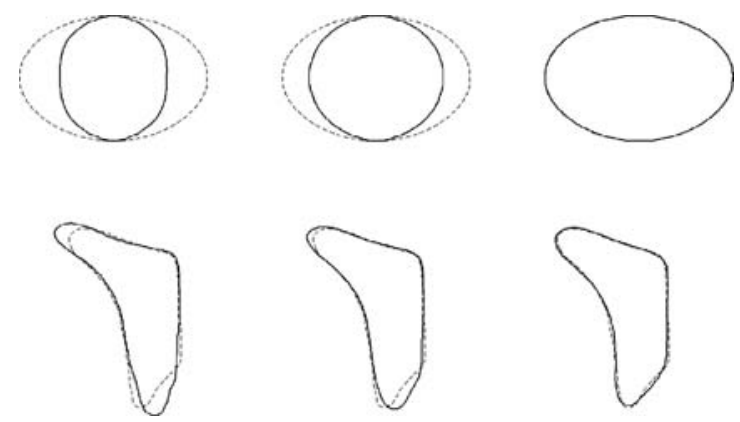

Figure 6. Minimization of $F_{\text {shape }}$ with the flow given in Eq. (20), $\phi$ and $\mathbf{x}_{T}$ being fixed. The prior shape is in solid line and the active contour in dotted line. The first row presents the shape evolution of the PCA model of 30 ellipses (see Section 2.2.2). The zero level set of the shape function $\hat{\phi}$ evolves to match with the active contour representing an ellipse taken in the training set. The second row shows the shape evolution of the PCA model of 45 left brain ventricles (see also Section 2.2.2). The shape model changes to match with the active contour representing a left brain ventricle taken in the training set.

where $\mathbf{e}_{\text {pca }}^{i}$ is the $i$ th principal component/eigenvector of the PCA presented in Section 2.2 and $\Omega_{\text {pca }}$ is the space of PCA variables defined by $\Omega_{\text {pca }}=$ $\left[-3 \lambda_{1}, 3 \lambda_{1}\right] \times \ldots \times\left[-3 \lambda_{p}, 3 \lambda_{p}\right]$ whose $\lambda_{i}$ is the eigenvalue of the $i$ th principal component. The evolution Eq. (11) changes the shape function $\hat{\phi}$ to match its zero level set with the active contour. Figure 6 presents this shape matching.

The flow minimizing $F_{\text {shape }}$ w.r.t. the vector of geometric transformations $\mathbf{x}_{T}$ is:

$$
\left\{\begin{array}{l}
d_{t} \mathbf{x}_{T}(t)=-2 \int_{0}^{1} \hat{\phi}\left\langle\nabla \hat{\phi}, \nabla_{\mathbf{x}_{T}} h_{\mathbf{x}_{T}}(C)\right\rangle\left|C^{\prime}\right| d p \\
\text { in }] 0, \infty\left[\times \Omega_{T},\right. \\
\mathbf{x}_{T}(t=0)=\mathbf{x}_{T_{0}} \text { in } \Omega_{T} .
\end{array}\right.
$$


In our work, we have considered the 2-D rigid (denoted by $h_{\mathbf{x}_{T}^{r}}$ ) and affine (denoted by $h_{\mathbf{x}_{T}^{a}}$ ) transformations:

$$
\begin{aligned}
& h_{\mathbf{x}_{T}^{r}}: x \rightarrow h_{(s, \theta, T)}(x)=s R_{\theta} x+T, \\
& h_{\mathbf{x}_{T}^{a}}: x \rightarrow h_{\left(s_{x}, s_{y}, \theta, s_{h}, T\right)}(x)=R_{s c} R_{\theta} R_{s h} x+T,
\end{aligned}
$$

where

$$
\begin{aligned}
& R_{s c}=\left(\begin{array}{ll}
s_{x} & 0 \\
0 & s_{y}
\end{array}\right), \quad R_{\theta}=\left(\begin{array}{ll}
\cos \theta & \sin \theta \\
-\sin \theta & \cos \theta
\end{array}\right), \\
& R_{s h}=\left(\begin{array}{ll}
1 & s_{h} \\
0 & 1
\end{array}\right) \quad \text { and } \quad T=\left(\begin{array}{c}
T_{x} \\
T_{y}
\end{array}\right) .
\end{aligned}
$$

The vector of rigid transformations $\mathbf{x}_{T}^{r}$ is composed of a scale parameter $s$, an angle of rotation $\theta$ and a vector of translations $T$ and the vector of affine transformations $\mathbf{x}_{T}^{a}$ is composed of two scale parameters $s_{x}$ in $x$-direction and $s_{y}$ in $y$-direction, an angle of rotation $\theta$, a shearing parameter $s_{h}$ and a vector of translations $T$. We will analyze the choice of the affine transformations in Section 5. Finally, the domain of the rigid/affine transformations is called $\Omega_{T}$.

As a consequence, the gradient term $\nabla_{\mathbf{x}_{T}} h_{\mathbf{x}_{T}}$ in Eq. (12) depending on geometric transformations is:

$$
\nabla_{\mathbf{x}_{T}^{r}} h_{\mathbf{x}_{T}^{r}}(x)=\left(\begin{array}{l}
\frac{\partial h_{\mathbf{x}_{T}^{r}}}{\partial s}(x)=R_{\theta} x \\
\frac{\partial h_{\mathbf{x}_{T}^{r}}}{\partial \theta}(x)=s \partial_{\theta} R_{\theta} x \\
\frac{\partial h_{\mathbf{x}_{T}^{r}}}{\partial T}(x)=\mathbf{1}
\end{array}\right),
$$

for 2-D rigid transformations and

$$
\nabla_{\mathbf{x}_{T}^{a}} h_{\mathbf{x}_{T}^{a}}(x)=\left(\begin{array}{l}
\frac{\partial h_{\mathbf{x}_{T}^{a}}}{\partial s_{x}}(x)=\left(\partial_{s_{x}} R_{s c}\right) R_{\theta} R_{s h} x \\
\frac{\partial h_{\mathbf{x}_{T}^{a}}}{\partial s_{y}}(x)=\left(\partial_{s_{y}} R_{s c}\right) R_{\theta} R_{s h} x \\
\frac{\partial h_{\mathbf{x}_{T}^{a}}}{\partial \theta}(x)=R_{s c}\left(\partial_{\theta} R_{\theta}\right) R_{s h} x \\
\frac{\partial h_{\mathbf{x}_{T}^{a}}}{\partial s_{h}}(x)=R_{s c} R_{\theta}\left(\partial_{s_{h}} R_{s h}\right) x \\
\frac{\partial h_{\mathbf{x}_{T}^{a}}}{\partial T}(x)=\mathbf{1}
\end{array}\right),
$$

for 2-D affine transformations. The evolution Eq. (12) realizes the rigid and affine registration between the zero level set of the shape model $\hat{\phi}$ and the active contour. Figures 7 and 8 present affine registrations.

Note that the function $\hat{\phi}$ is evaluated at $\left(\mathbf{x}_{\mathrm{pca}}\right.$, $h_{\mathbf{x}_{T}}(C(q))$ in Eqs. (10)-(12).

Let us now express the previous equations in a variational level set formulation as presented in Zhao et al. (1996) and Chen et al. (2002). The level set approach of Zhao et al. (1996), rather than (Caselles et
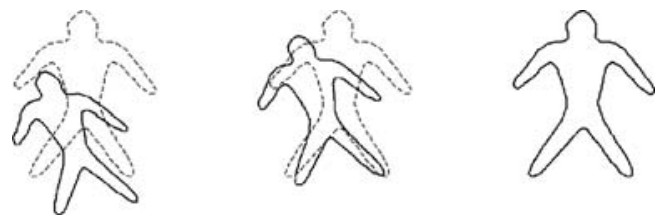

Figure 7. Minimization of $F_{\text {shape }}$ with the flow given in Eq. (12), $\phi$ and $\mathbf{x}_{\mathrm{pca}}$ being fixed. The row of images represents the affine registration of a prior shape in solid line into an active contour in dotted line.
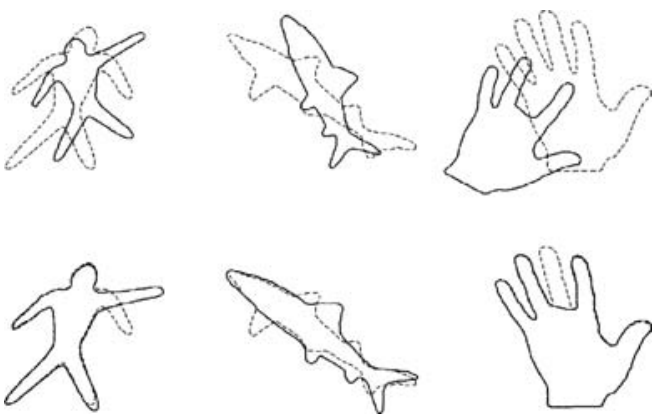

Figure 8. Minimization of $F_{\text {shape }}$ with the flow given in Eq. (12), $\phi$ and $\mathbf{x}_{\text {pca }}$ being fixed. Each column presents the affine registration of a prior shape in solid line into an active contour in dotted line. The first row shows the initial position of the shapes and the second row the registered shapes. This registration process works with shapes having different local structures and missing information.

al., 1997; Kichenassamy et al., 1996), will be used to prove the existence of solution minimizing our energy functional in the space of functions with bounded variation. The level set formulation of the shape functional from Eq. (6) is:

$$
F_{\text {shape }}=\int_{\Omega} \hat{\phi}^{2}\left(\mathbf{x}_{\text {pca }}, h_{\mathbf{x}_{T}}(x)\right)|\nabla \varphi| \delta(\varphi) d \Omega
$$

where $\varphi$ is a level set function embedding the active contour $C, \delta(\cdot)$ is the Dirac function and $\delta(\varphi)$ is the contour measure on $\{\varphi=0\}$. The level set formulation of $F_{\text {shape }}(18)$ is equivalent to the geometric formulation (6) because of the Coarea formula (Evans and Gariepy, 1992) which proves that $L_{f}(\varphi)=\int_{\Omega} f(x) \delta(\varphi)|\nabla \varphi| d x$ $=\oint_{0}^{L} f(C(s)) d s=\oint_{0}^{1} f(C(q))\left|C^{\prime}(q)\right| d q$ where $L$ is the length of $C=\{\varphi=0\}$. The proof is presented in Samson et al. (2000). 
The level set formulation of Eqs. (10)-(12) are:

$$
\begin{gathered}
\left\{\begin{aligned}
& \partial_{t} \varphi(t, x)=\left(\hat{\phi}^{2} \kappa-\left\langle\nabla \hat{\phi}^{2}, \frac{\nabla \varphi}{|\nabla \varphi|}\right\rangle\right) \delta(\varphi) \\
&\text { in }] 0, \infty[\times \Omega, \\
& \varphi(0, x)=\varphi_{0}(x) \text { in } \Omega, \\
& \frac{\delta(\varphi)}{|\nabla \varphi|} \partial_{\mathcal{N}} \varphi=0 \text { on } \partial \Omega,
\end{aligned}\right. \\
\left\{\begin{array}{c}
d_{t} \mathbf{x}_{\mathrm{pca}}(t)=-2 \int_{\Omega} \hat{\phi} \nabla_{\mathbf{x}_{\mathrm{pca}}} \hat{\phi}|\nabla \varphi| \delta(\varphi) d \Omega \\
\text { in }] 0, \infty\left[\times \Omega_{\mathrm{pca}},\right. \\
\mathbf{x}_{\mathrm{pca}}(t=0)=\mathbf{x}_{\mathrm{pca}_{0}} \text { in } \Omega_{\mathrm{pca}},
\end{array}\right. \\
\left\{\begin{array}{c}
d_{t} \mathbf{x}_{T}(t)=-2 \int_{\Omega} \hat{\phi}\left\langle\nabla \hat{\phi}, \nabla_{\mathbf{x}_{T}} h_{\mathbf{x}_{T}}\right\rangle|\nabla \varphi| \delta(\varphi) d \Omega \\
\text { in }] 0, \infty\left[\times \Omega_{T},\right.
\end{array}\right. \\
\mathbf{x}_{T}(t=0)=\mathbf{x}_{T_{0}} \text { in } \Omega_{T} .
\end{gathered}
$$

In our segmentation model, the flows given by the Eqs. (19)-(21) are simultaneously used to constraint the active contour to get a shape of interest whatever the position of the active contour in the image.

In a nutshell, we have defined in this section a process to force the active contour to get a particular shape. In the next section, we will introduce image information in our segmentation method to capture the object of interest in the image.

\subsection{The Region Term $F_{\text {region }}$}

In this section, we define a functional to drive the shape model towards a homogeneous intensity region with the shape of interest. If our objects of interest are supposed to have a smooth intensity surface then the MumfordShah (MS) model is the most adapted model to segment these objects.

At this stage, we had the choice to apply the MS model either on the active contour or the shape prior. Since the MS method applied on the active contour will extract globally homogeneous regions (Vese and Chan, 2002) and our objective is to capture an object belonging to a given shape space then the best solution is to apply the MS-based force on the shape prior. Indeed, this new force will globally drive the shape prior towards a homogeneous intensity region with the shape of interest. An illustration of this choice will appear in Section 4.2.2.
We have modified the Mumford-Shah functional (Mumford and Shah, 1989) presented by Vese and Chan (2002) to segment a smooth region whose shape is described by the PCA model:

$$
\begin{aligned}
& F_{\text {region }}\left(\mathbf{x}_{\mathrm{pca}}, \mathbf{x}_{T}, u_{\mathrm{in}}, u_{\mathrm{out}}\right)= \\
& \oint_{\hat{C}\left(\mathbf{x}_{\mathrm{pca}}, \mathbf{x}_{T}\right)} d s+\int_{\Omega_{\mathrm{in}}\left(\mathbf{x}_{\mathrm{pca}}, \mathbf{x}_{T}\right)}\left(\left|I-u_{\mathrm{in}}\right|^{2}+\mu\left|\nabla u_{\mathrm{in}}\right|^{2}\right) d \Omega \\
& +\int_{\Omega_{\text {out }}\left(\mathbf{x}_{\mathrm{pca}}, \mathbf{x}_{T}\right)}\left(\left|I-u_{\mathrm{out}}\right|^{2}+\mu\left|\nabla u_{\mathrm{out}}\right|^{2}\right) d \Omega
\end{aligned}
$$

where the curve $\hat{C}$ is the zero level set of the shape function $\hat{\phi}$ extracted from the PCA process. The function $\hat{\phi}$ defines an image partitioned into two regions $\Omega_{\text {in }}$ and $\Omega_{\text {out }}$, representing respectively the object and the background, whose common boundary is $\hat{C}$ :

$$
\left\{\begin{array}{l}
\Omega_{\mathrm{in}}\left(\mathbf{x}_{\mathrm{pca}}, \mathbf{x}_{T}\right)=\left\{x \in \Omega \mid \hat{\phi}\left(x, \mathbf{x}_{\mathrm{pca}}, \mathbf{x}_{T}\right)>0\right\}, \\
\Omega_{\mathrm{out}}\left(\mathbf{x}_{\mathrm{pca}}, \mathbf{x}_{T}\right)=\left\{x \in \Omega \mid \hat{\phi}\left(x, \mathbf{x}_{\mathrm{pca}}, \mathbf{x}_{T}\right)<0\right\}, \\
\hat{C}\left(\mathbf{x}_{\mathrm{pca}}, \mathbf{x}_{T}\right)=\left\{x \in \Omega \mid \hat{\phi}\left(x, \mathbf{x}_{\mathrm{pca}}, \mathbf{x}_{T}\right)=0\right\} .
\end{array}\right.
$$

The minimization of $F_{\text {region }}$ determines the shape parameters $\mathbf{x}_{\mathrm{pca}}$ and the parameters $\mathbf{x}_{T}$ of the rigid or affine transformation of the contour $\hat{C}$ which captures a region having the shape of interest. In our work, we have not considered the smoothing term, $\oint_{\hat{C}} d s$, since shapes generated by the PCA are smooth enough. The functional $F_{\text {region }}$ can be written with the shape function $\hat{\phi}$ :

$$
\begin{aligned}
& F_{\text {region }}\left(\mathbf{x}_{\mathrm{pca}}, \mathbf{x}_{T}, u_{\text {in }}, u_{\text {out }}\right) \\
& =\int_{\Omega} \Theta_{\text {in }} H\left(\hat{\phi}\left(\mathbf{x}_{\mathrm{pca}}, \mathbf{x}_{T}\right) d \Omega\right. \\
& \quad+\int_{\Omega} \Theta_{\text {out }} H\left(-\hat{\phi}\left(\mathbf{x}_{\mathrm{pca}}, \mathbf{x}_{T}\right) d \Omega,\right.
\end{aligned}
$$

where $H(\cdot)$ is the Heaviside function, $\Theta_{r}=\left|I-u_{r}\right|^{2}+$ $\mu\left|\nabla u_{r}\right|^{2}$ and $r=$ in or out.

The modified MS functional (24) is minimized using the gradient descent method for $\mathbf{x}_{\mathrm{pca}}$ and $\mathbf{x}_{T}$ and solving the Euler-Lagrange equations for $u_{\text {in }}$ and $u_{\text {out }}$ :

$$
\left\{\begin{aligned}
& d_{t} \mathbf{x}_{\mathrm{pca}}(t)=\int_{\Omega}\left(\Theta_{\text {in }}-\Theta_{\text {out }}\right) \frac{\partial \hat{\phi}}{\partial \mathbf{x}_{\mathrm{pca}}} \delta(\hat{\phi}) d \Omega, \\
&=\int_{\Omega}\left(\Theta_{\text {in }}-\Theta_{\text {out }}\right) \nabla_{\mathbf{x}_{\mathrm{pca}}} \hat{\phi} \delta(\hat{\phi}) d \Omega, \\
&\text { in }] 0, \infty\left[\times \Omega_{\mathrm{pca}},\right. \\
& \mathbf{x}_{\mathrm{pca}}(t=0)=\mathbf{x}_{\mathrm{pca}_{0}} \text { in } \Omega_{\mathrm{pca}},
\end{aligned}\right.
$$



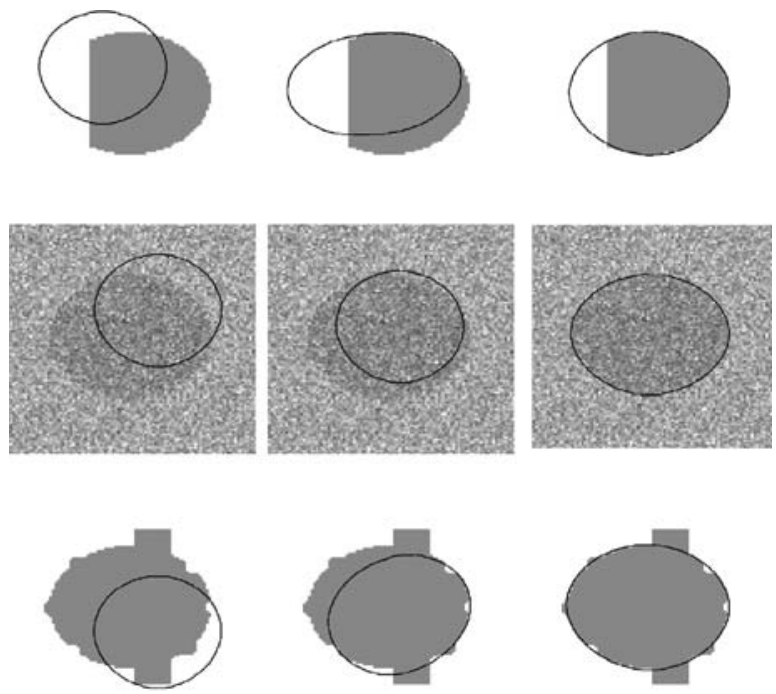

(i)

Figure 9. Minimization of $F_{\text {region }}$ with the flows given by Eqs. (25)(27). The first row presents the evolution of the segmentation process of an ellipse partially cut. The second row shows the segmentation of a noisy ellipse. And the third row is the segmentation of an occluded ellipse.

$$
\left\{\begin{array}{c}
d_{t} \mathbf{x}_{T}(t)=\int_{\Omega}\left(\Theta_{\text {in }}-\Theta_{\text {out }}\right) \frac{\partial \hat{\phi}}{\partial \mathbf{x}_{T}} \delta(\hat{\phi}) d \Omega, \\
=\int_{\Omega}\left(\Theta_{\text {in }}-\Theta_{\text {out }}\right) \\
\left.\left\langle\nabla \hat{\phi}, \nabla_{\mathbf{x}_{T}} h_{\mathbf{x}_{T}}\right\rangle \delta(\hat{\phi}) d \Omega, \text { in }\right] 0, \infty\left[\times \Omega_{T},\right. \\
\mathbf{x}_{T}(t=0)=\mathbf{x}_{T_{0}} \text { in } \Omega_{T},
\end{array}\right.
$$

$$
\left\{\begin{aligned}
& \partial_{t} u_{\text {in }}(t, x)= u_{\text {in }}-I-\mu \Delta u_{\text {in }} \\
&\text { in }] 0, \infty[\times\{\hat{\phi}>0\}, \\
& u_{\text {in }}(0, x)=I \text { in }\{\hat{\phi}>0\}, \\
& \partial_{t} u_{\text {out }}(t, x)=u_{\text {out }}-I-\mu \Delta u_{\text {out }} \\
&\text { in }] 0, \infty[\times\{\hat{\phi}<0\}, \\
& u_{\text {out }}(0, x)=I \text { in }\{\hat{\phi}<0\} .
\end{aligned}\right.
$$

Figure 9 shows that the minimization of $F_{\text {region }}$ segments objects of interest when a part of information is missing and in presence of noise and occlusion. This model can also be used to segment the left brain ventricle on Fig. 10.

However, this segmentation method can not handle local structure variations (see Fig. 9(i)) when e.g. an ellipse presents irregular boundaries. The model has not captured the local edge variations since it only

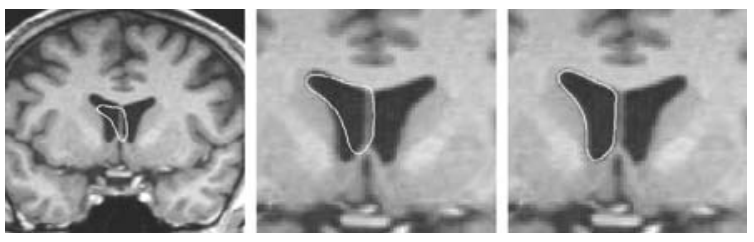

Figure 10. Segmentation of the left ventricle with the flows given by Eqs. (25)-(27).

deals with global shape variations provided by the PCA model. If we want to be able to capture the local variations around the global shape we found, we need to add a local criteria to our energy functional. We will consider for this purpose the classic geodesic active contour given by $F_{\text {boundary }}$.

We noticed that another segmentation method based on the Mumford-Shah functional and the PCA model of Leventon et al. has been proposed by Tsai et al. (2001) but for a reduced model of the MS model. Indeed, they have employed the piecewise constant approximation of the MS functional proposed by Chan and Vese (2001) to define a functional equal to Eq. (22) when $\mu=0$. Our model consider the general model of the MS model, i.e. the piecewise smooth approximation, as introduced by Vese and Chan (2002) in the context of active contours. We think that the piecewise smooth case of the MS model allows us to decrease the intensity bias present in the piecewise constant case. This bias, which can significantly affect the computation of the parameters $\mathbf{x}_{\mathrm{pca}}$ and $\mathbf{x}_{T}$, is due to the the inhomogeneity of the outside region, i.e. the background, with respect to the inside region, the object of interest. The reason is that the general MS model considers the grey value information by averaging locally the intensities in a neighborhood (whose size depends on $\mu$, Eq. (33)) surrounding the contour by means of diffusion whereas the reduced MS model considers the grey value information by averaging globally the intensities over the background region and the object region. Cremers et al. (2002) also compared the two cases of the MS functional and they noticed that the segmentation results are less accurate with the reduced model of MS because of the same reasons.

\subsection{Combining Shape-Based, Boundary-Based and Region-Based Functionals}

In Section 3.2, we have studied a shape-based functional $F_{\text {shape }}$ that evaluates the similarity between the active contour shape and the object shape prior to be 
segmented. In Section 3.3, we have analyzed a regionbased functional $F_{\text {region }}$ which allows us to drive globally the shape prior towards a homogeneous intensity region. We now combine these two functionals with the boundary-based functional $F_{\text {boundary }}$ which captures the object edges to obtain a functional $F$ (see Eq. (5)) to segment objects with a shape model and with global and local image information.

The energy minimization of $F$ is performed using the calculus of variations and the gradient descent method. We obtain a system of coupled evolution equations whose steady-state solution gives the minimum of $F$, which means the solution of the segmentation problem. The existence of a minimum of $F$ is proved in annex. The functional $F$ is expressed in the Eulerian/level set framework as follows:

$$
\begin{aligned}
F & =\int_{\Omega} f\left(x, \mathbf{x}_{\mathrm{pca}}, \mathbf{x}_{T}\right)|\nabla \varphi| \delta(\varphi) d \Omega \\
& +\beta_{r} \int_{\Omega}\left(\Theta_{\mathrm{in}} H\left(\hat{\phi}\left(\mathbf{x}_{\mathrm{pca}}, \mathbf{x}_{T}\right)\right)+\Theta_{\text {out }} H(-\hat{\phi})\right) d \Omega
\end{aligned}
$$

whose

$$
f\left(x, \mathbf{x}_{\mathrm{pca}}, \mathbf{x}_{T}\right)=\beta_{s} \hat{\phi}^{2}\left(\mathbf{x}_{\mathrm{pca}}, h_{\mathbf{x}_{T}}(x)\right)+\beta_{b} g(|\nabla I(x)|) .
$$

And the evolution equations minimizing $F$ are:

$$
\begin{aligned}
& \left\{\begin{aligned}
& \partial_{t} \varphi(t, x)=\left(f \kappa-\left\langle\nabla f, \frac{\nabla \varphi}{|\nabla \varphi|}\right\rangle\right) \delta(\varphi) \\
&\text { in }] 0, \infty[\times \Omega, \\
& \varphi(0, x)=\varphi_{0}(x) \text { in } \Omega \\
& \frac{\delta(\varphi)}{|\nabla \varphi|} \partial_{\mathcal{N} \varphi}=0 \text { on } \partial \Omega
\end{aligned}\right. \\
& \left\{\begin{array}{c}
d_{t} \mathbf{x}_{\mathrm{pca}}(t)=-\int_{\Omega} \nabla_{\mathbf{x}_{\mathrm{pca}}} \hat{\phi}\left(2 \beta_{s} \hat{\phi}|\nabla \varphi| \delta(\varphi)+\right. \\
\left.\left.\beta_{r}\left(\Theta_{\text {in }}-\Theta_{\text {out }}\right) \delta(\hat{\phi})\right) d \Omega \text { in }\right] 0, \infty\left[\times \Omega_{\mathrm{pca}},\right. \\
\mathbf{x}_{\mathrm{pca}}(t=0)=\mathbf{x}_{\mathrm{pca}_{0}} \text { in } \Omega_{\mathrm{pca}},
\end{array}\right. \\
& \left\{\begin{array}{l}
d_{t} \mathbf{x}_{T}(t)=-\int_{\Omega}\left\langle\nabla \hat{\phi}, \nabla_{\mathbf{x}_{T}} h_{\mathbf{x}_{T}}\right\rangle \quad\left(2 \beta_{s} \hat{\phi}|\nabla \varphi| \delta(\varphi)\right. \\
\left.\left.\quad+\beta_{r}\left(\Theta_{\text {in }}-\Theta_{\text {out }}\right) \delta(\hat{\phi})\right) d \Omega \text { in }\right] 0, \infty\left[\times \Omega_{T},\right. \\
\mathbf{x}_{T}(t=0)=\mathbf{x}_{T_{0}} \text { in } \Omega_{T},
\end{array}\right.
\end{aligned}
$$

$$
\left\{\begin{aligned}
& \partial_{t} u_{\text {in }}(t, x)= u_{\text {in }}-I-\mu \Delta u_{\text {in }} \\
&\text { in }] 0, \infty[\times\{\hat{\phi}>0\}, \\
& u_{\text {in }}(0, x)=I \text { in }\{\hat{\phi}>0\}, \\
& \partial_{t} u_{\text {out }}(t, x)=u_{\text {out }}-I-\mu \Delta u_{\text {out }} \\
&\text { in }] 0, \infty[\times\{\hat{\phi}<0\}, \\
& u_{\text {out }}(0, x)=I \text { in }\{\hat{\phi}<0\} .
\end{aligned}\right.
$$

\section{Experimental Results}

\subsection{Implementation Issues}

Concerning the PCA, the first stage consists in aligning rigidly the training curves representing the object of interest. This is realized using the shape similarity measure introduced by Chen et al. (2002):

$$
\begin{aligned}
a\left(C_{1}, C_{j}^{\text {new }}\right) & =\text { area of }\left(A_{1} \cup A_{j}^{\text {new }}\right. \\
& \left.-A_{1} \cap A_{j}^{\text {new }}\right) \quad \text { for } 2 \leq j \leq n,
\end{aligned}
$$

where $A_{1}$ and $A_{j}^{\text {new }}$ denote respectively the interior regions of the curves $C_{1}$ and $C_{j}^{\text {new }}$ where $C_{j}^{\text {new }}$ is the resulting curve from the rigid registration such that $C_{j}^{\text {new }}=s_{j} R_{\theta_{j}} C_{j}+T_{j}$ and $n$ is the number of training curves. $C_{1}$ and $C_{j}$ are aligned when the measure $a$ is minimized for the appropriate values $s_{j}^{\star}, \theta_{j}^{\star}$ and $T_{j}^{\star}$. These values are obtained by a global optimization algorithm called the genetic algorithm (Davis, 1991).

The second stage of the PCA consists in doing the singular values decomposition on the SDFs of the aligned training curves using the code provided by $\mathrm{Nu}$ merical Recipes (Numerical Recipies in $\mathrm{C}++$ and $\mathrm{C}$ ) on the matrix $\Sigma^{\text {dual }}=\frac{1}{n} M^{\top} M$ (see Section 2.2 for notations) to extract the $n$ eigenvalues $\mathbf{e}_{\text {pca }}^{i \text {,dual }}$ and the eigenvectors $\lambda_{\mathrm{pca}}^{i, \text { dual }}$. Note that the PCA is performed on $\Sigma^{\text {dual }}$ rather than $\Sigma$ to give faster and more accurate results. The eigenvectors $\mathbf{e}_{\mathrm{pca}}^{i}$ and the eigenvalues $\lambda_{\text {pca }}^{i}$ are then given by $\mathbf{e}_{\mathrm{pca}}^{i}=M \mathbf{e}_{\mathrm{pca}}^{i, \text { dual }}$ and $\lambda_{\text {pca }}^{i}=\lambda_{\text {pca }}^{i, \text { dual }}$.

The evolution Eqs. (30) to (33) are numerically solved by iterating the following stages until convergence is reached:

1. Computation of the shape function $\hat{\phi}\left(\mathbf{x}_{\mathrm{pca}}, \mathbf{x}_{T}\right)$ using Eq. (4) and performing the rigid and affine transformations (scaling, rotation, translations and 
shearing) with the $B$-splines interpolation method (Unser, 1999).

2. Calculation of the gradient $\nabla \hat{\phi}$ using a central difference scheme. The term $\nabla_{\mathbf{x}_{\mathrm{pca}}} \hat{\phi}$ is given by the eigenvectors of the PCA model and $\nabla_{\mathbf{x}_{T}} h_{\mathbf{x}_{T}}$ is computed according to Eqs. (16) and (17).

3. Discretization of terms $|\nabla \varphi|$ and $\left\langle\nabla f, \frac{\nabla \varphi}{|\nabla \varphi|}\right\rangle$ with the Osher-Sethian numerical scheme (Osher and Sethian, 1988). Computation of the curvature with central difference schemes. The Dirac function $\delta$ and the Heaviside function $H$ are computed by slightly regularized versions following (Zhao et al., 1996; Chen et al., 2002).

4. Functions $u_{\text {in }}$ and $u_{\text {out }}$ are computed in $\{\hat{\phi}>0\}$ and $\{\hat{\phi}<0\}$ with the method proposed in Vese and Chan (2002).

5. Eqs. (30)-(33) are computed each iteration in the following order: (33), (32), (31) and (30). However, we noticed that the order of the gradient descents has no effect on the solution.

6. Re-distancing the level set function at every iteration with the Fast Marching Method of Adalsteinsson and Sethian (1995).

\subsection{Results}

4.2.1. Synthetic Images. In this first experiment, we considered an ellipse partially occluded with a noisy boundary and an ellipse whose part of the shape is missing. We applied the PCA on the training set of 30 ellipses as proposed in Section 2.2.2. The alignment process, which performs the registration of shapes by genetic programming, needed around 1 minute per $128 \times 128$ ellipse. Even if the alignment step is not fast because the genetic optimization is a global optimization procedure, it is done once for all experiments. The rest of the PCA is fast. The decomposition into principal components, which is also done only once for all experiments, took a few seconds. And the most important procedures (since they are used at each iteration) are very fast: the computation of a new shape according to Eq. (4) (with an arbitrary vector $\mathbf{x}_{\mathrm{pca}}$ ) took around $10^{-3}$ second and the spatial transformations done with the B-splines programming also needed around $10^{-3}$ second. In all the following experiments, from Fig. 11 to Fig. 16, the segmentation including shape and pose parameters took around 5 minutes.

We firstly used our segmentation model to segment an ellipse with irregular boundaries which is partially occluded by a vertical bar. Figure 11 presents a geodesic
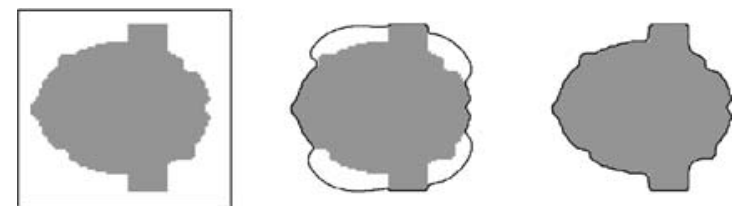

Figure 11. Evolution of the geodesic active contour without shape prior information.
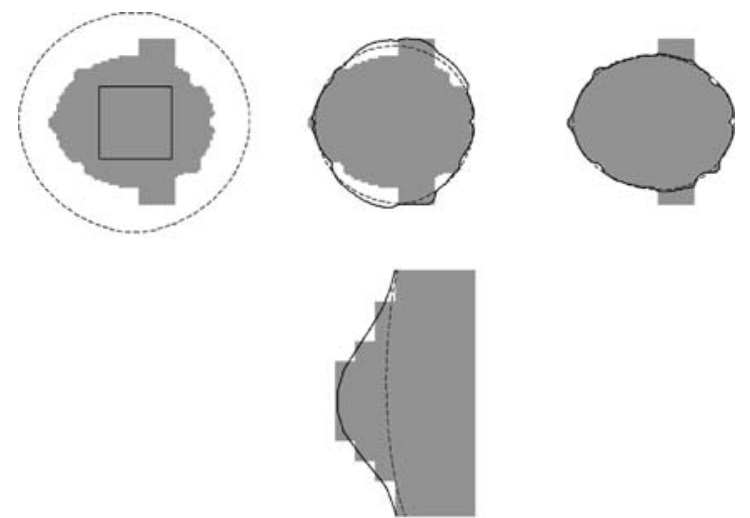

(d)

Figure 12. The first row presents the evolution of the active contour (in solid line) with a shape prior (in dotted line). In the second row, we zoom on the left point of the ellipse to show that our model is able to capture local deformations around the shape prior.

active contour without a shape prior and Figs. 12 and 13 with a shape prior by taking $\beta_{s}=1 / 3, \beta_{b}=1$, $\beta_{r}=10, \mu=50$ and $\Delta t=0.1$.

The way of choosing the weighting parameters is as follows. $\beta_{b}$ is always equal to $1 . \mu$ determines the size of the neighborhood where the grey value information is averaged by means of diffusion (33). Then, $\beta_{r}$ is chosen such that the shape prior is attracted toward the region to be segmented. Finally, $\beta_{s}$ is elected in order to allow the active contour to move around the shape prior in order to capture local boundaries. The active contours on Figs. 12 and 13 have captured high image gradients, i.e. the boundary variations (see Fig. 12(d)), and also handled the problem of occlusion thanks to the information contained in the prior shape model.

In the second example, our extraction model is applied to extract an ellipse which is partially cut. Figure 14 presents a geodesic active contour without a shape prior and Figs. 15 and with a shape prior by taking $\beta_{s}=1 / 3, \beta_{b}=1, \beta_{r}=10, \mu=50$ and $\Delta t=0.1$. The active contour on Fig. 15 has captured high image gradients and also the missing part thanks to the information contained in the prior shape model. Figure 16 

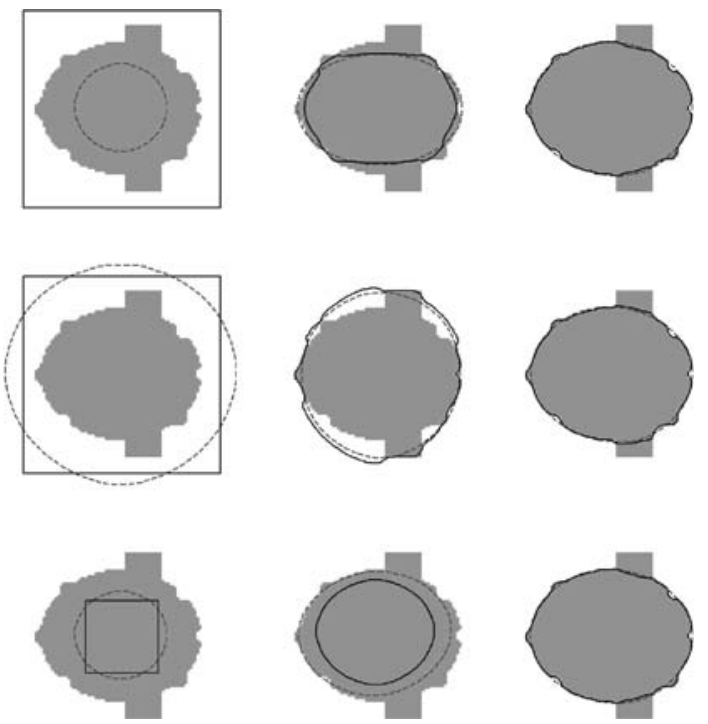

Figure 13. Our active contour model (in solid line) with a shape prior (in dotted line) is robust w.r.t. an initial active contour and an initial shape prior outside or inside the ellipse.
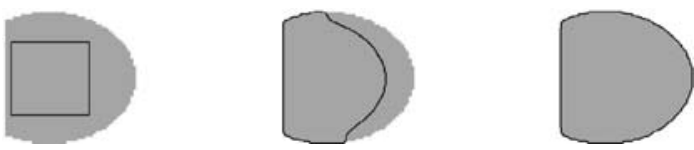

Figure 14. Evolution of the geodesic active contour without shape prior information.
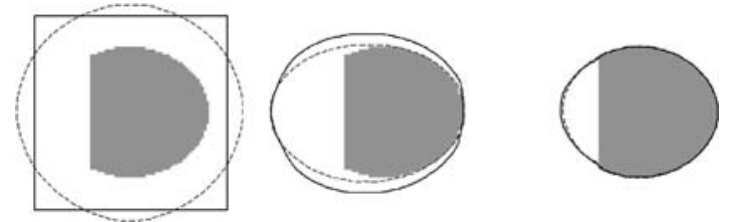

Figure 15. Evolution of the active contour (in solid line) with the shape prior (in dotted line).

illustrates what happens when only the mean shape $\bar{\phi}$ in the PCA model (Eq. (4)) is used. In this experiment, the eigenmodes of variation are essential to get a satisfactory result.

Thus, our shape-based active contour model can segment objects with missing information, occlusion and local shape variations.

4.2.2. Medical Image. In this second experiment, we considered the left brain ventricle and its training set of 45 shapes. The alignment process, done by genetic

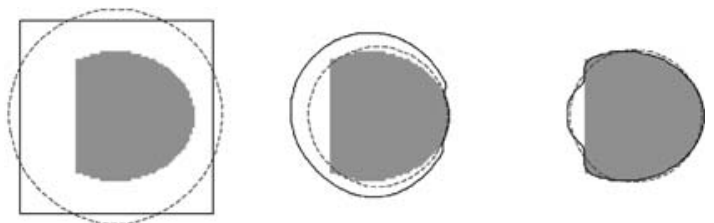

Figure 16. Result obtained using only the mean shape $\bar{\phi}$ in the PCA model (Eq. (4)) without the eigenmodes of variation.
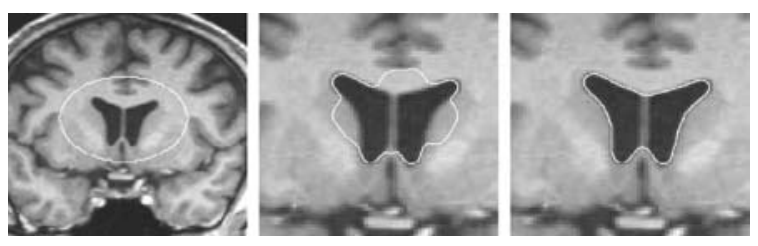

Figure 17. Evolution of the geodesic active contour without shape prior information.
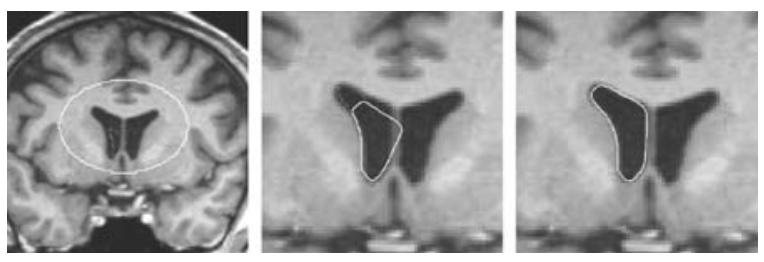

Figure 18. Evolution of the active contour (in solid line) with a shape prior (in dotted line).

programming, took around 1 minute per $128 \times 128$ ventricle. The decomposition into principal components needed a few seconds and, as in the case of ellipses described in the previous section, the computation of a new shape according to Eq. (4) took around $10^{-3} \mathrm{sec}-$ ond and the spatial transformations done with the Bsplines programming around $10^{-3}$ second. In the experiments, from Figs. 19 to 23, the segmentation including shape and pose parameters took around 5 minutes.

We have used our segmentation model to capture the left brain ventricle. Fig. 17 presents the evolving geodesic active contour without a shape prior and Fig. 18 with a shape prior by choosing $\beta_{s}=2, \beta_{b}=1$, $\beta_{r}=100, \mu=50$ and $\Delta t=0.1$.

We observe on Fig. 18 that the active contour has well captured the left ventricle whereas the initial contour was around the two ventricles (see Fig. 18). This segmentation result could not be obtained without a shape prior with the same initial contour as shown on Fig. 17. The segmentation model has also provided the shape of the model which best fits the ventricle lying in the image. 

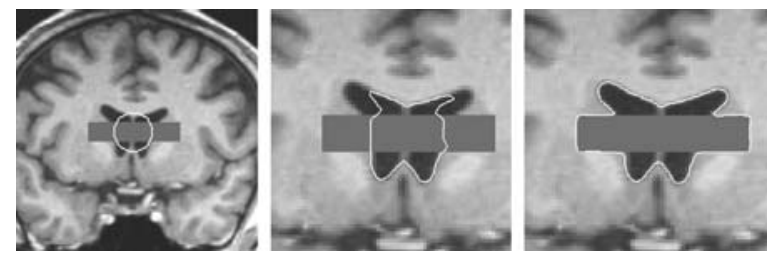

Figure 19. Evolution of the region-based active contour of Vese and Chan (2002) in the presence of an occlusion.
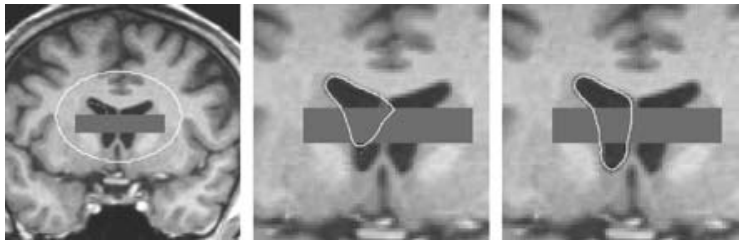

Figure 20. Evolution of the active contour (in solid line) with a shape prior (in dotted line) in the presence of an occlusion.
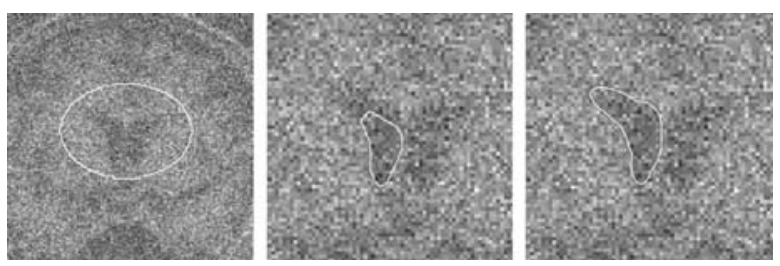

Figure 21. Evolution of the active contour (in solid line) with a shape prior (in dotted line) in the presence of an important quantity of noise.

Figure 18 illustrates the Section 3.3 remark: in this case, the Mumford-Shah model applied on the active contour will separate both ventricles (that form a homogeneous intensity region) from the rest of the white matter. The shape force will be then opposed to the region force since the shape force will pull the active contour inside the right ventricle towards the left ventricle whereas the Mumford-Shah force will constrain the active contour to stay on the border of ventricles. Our model avoids this situation since region-based forces are only applied on the contour of the shape prior and not on the active contour itself.

For Figs. 19, 20 and 21, we followed the experiments done by Cremers et al. (2002). Indeed, we added an occlusion bar on Figs. 19 and 20 and an important amount of noise on Fig. 21. Figure 19 shows the evolution of the region-based active contour of Chan and Vese (2002) which fails to segment the left ventricle.
Hence, a shape-based term is essential to successfully segment the ventricle as shown on Figs. 20 and 21.

\section{Discussion}

The active contour obtained from the minimization of the energy functional defined in Eq. (5) is able to capture high image gradients and a homogeneous intensity region whose shape matches the object of interest. We have seen on Figs. 15 and 12 that the shape information allows us to solve the problems of missing information/occlusion while being sensitive to local shape variations. Indeed, small deformations are allowed around the zero level set of the shape function on a distance that depends on the relative weight $\beta_{s} / \beta_{b}$. These complex deformations are easier to handle in the level set framework, thanks to its intrinsic representation, than parametric ones (Cremers et al., 2002; Cremers et al., 2002b).

As we mentioned previously, the proposed segmentation model can be seen either as an extension of the model of Chen et al. (2002) where we have introduced the shape model of Leventon et al. (2000) and the Mumford-Shah model (Vese and Chan, 2002) or as an energy formulation of the model of Leventon et al. with the MS energy functional. Using the variational formulation of Chen et al. enables us to prove the existence of a solution minimizing our energy functional in the space of functions with bounded variation (see Appendix).

Note that the region term based on the MumfordShah functional increases the speed of convergence towards the solution and it also improves the robustness of the model w.r.t. the initial condition, noise and complex background.

The PCA shape model we use in our segmentation method presents a good compromise when compared to other models. First, the computation of the $p$ principal components which are orthonormal basis functions is straightforward and fast, using the singular values decomposition method. These functions are then used to produce new shapes of the object of interest according to a simple linear equation. The number $p$ of principal components, i.e. the number of the shape model parameters, is often small as we have noticed for the ellipse (see Fig. 1) that needs only one principal component or for the left brain ventricle (see Fig. 3) with three principal components. Thus, global shape variations are modeled by a small number of variables which greatly reduces the complexity of the problem, when 
compared e.g. to Paragios et al. model (Paragios et al., 2003). Indeed, their shape model generates more complex shapes than the PCA but the $p$ shape parameters of the PCA model is replaced in their work by a local deformation field to be evaluated on a $\delta$-band around the zero level set of the shape function. Note that the shapes produced by the PCA are obviously implicit and intrinsic, i.e. independent of the parametrization, which facilitates the morphing and the registration processes. However, shape functions provided by the PCA are not exactly SDFs as proved by Leventon (2000). Nevertheless, shape functions of the PCA are very close to SDFs, which allow us to use them in practice. The same remark holds for a spatial transformation applied on the shape function. Indeed, the shape function after an affine transformation is (in general) not a distance function. Thus, the family of shapes obtained by affine transformations is no longer an equivalent class w.r.t. Eq. (9) and the shape term (6). The previous observations lead to the following condition to successfully use the morphing and the registration processes: a point belonging to the shape function constructed by the PCA or computed by a spatial transformation must see its height continuously decreasing when moving towards the zero level set even if its gradient is not exactly in the normal direction of the zero level set. Fortunately, we experimentally noticed that all shape functions generated by the PCA and changed by an affine transformation satisfy this condition.

The shape functions given by the PCA are thus not accurate SDFs but there are two ways to obtain exact SDFs (and have a strict equality in Eq. (9)). Either the shape function is projected in the SDFs space by re-distancing $\hat{\phi}$ as a SDF or the framework of Charpiat et al. (2003) can be used to define a mean and principal modes of variation for distance functions. In our segmentation model, we have to compute the transformation and shape parameters. However, Cremers et al. (2002,b) have defined two shape energies independent of the rigid transformations and the shape parameters. This means that their segmentation model had not to compute the vector of the rigid transformations $\mathbf{x}_{T}$ and the vector of shape parameters $\mathbf{x}_{\text {pca }}$ with Eqs. (31) and (32) since the correct pose parameters are automatically estimated and the shape parameters can be extracted by projection on the respective eigenmodes. Thus, is it really useful to estimate the registration parameters $\mathbf{x}_{T}$ and the shape parameters $\mathbf{x}_{\mathrm{pca}}$ ? It depends on two questions: does the current application need to compute transforma- tion and shape parameters and are affine or non-rigid transformations necessary? If the answer is positive for one of these questions, the estimation of these parameters will be imperative. In Chen et al. (2002) and Biswal and Hyde (1997) for example, the transformation parameters are used to align time series images in order to minimize the effect of motion on the fMRI signal.

Using a variational framework and the PDEs attached to it, we can consider other models such as Jehan-Besson et al. (2003) to segment objects by linearly combining energy functionals or the PDEs directly.

Recently and posterior to our publications (Bresson et al., 2003) and (Bresson et al., 2004), Rousson, Paragios and Deriche (2004) presented a model to extract structures of interest similar to ours. Indeed, they also proposed to integrate the implicit shape prior of Leventon et al. (2000) in a variational level set framework to derive two minimizing flows on the level set function representing the active contour and on the spatial transformations. Finally, the shape vector is computed by solving a linear system. We notice that the shape-based functional of Rousson et al. is similar to ours because it corresponds to the sum of square differences (SSD) between the active contour and the zero level set of the implicit shape prior. Indeed, their shapebased functional minimizes the difference between the level set function embedding the active contour and the level set-based shape prior weighted by the Dirac function applied to the level set function of the active contour. The difference between both works is the region-based functional. Rousson et al. proposed to add the model of geodesic active regions to the shape-based functional. However, as explained in Section 3.3, since our objective is to capture an object belonging to a given shape space then we think that the best solution is to apply directly the region-based force on the contour of the shape prior as we did.

Concerning the computation of the shape vector $\mathbf{x}_{\mathrm{pca}}$, i.e. the mode weights of the PCA. The computation of the mode weights is probably more efficient with Rousson et al. than ours since their technique provides the optimal mode weights at each iteration which minimizes the shape difference between the active contour and the shape prior. Our model does not directly provide the optimal mode weights at each iteration since it is a gradient descent flow, i.e. a local minimization technique. However, at the end of the segmentation/registration process, our technique will provide 
the optimal mode weights which minimizes the difference between the active contour shape and the prior shape. Moreover, the way of computing the $\mathbf{x}_{\mathrm{pca}}$ in the approach of Rousson et al. (2004) is given by an independent stage (solving a linear system) of the minimization energy functional w.r.t. $\mathbf{x}_{\mathrm{pca}}$ which means that no mathematical theories can be applied to prove the existence of a solution by opposition to our approach (see Appendix).

\section{Conclusion}

In this paper, we have proposed a new variational method to solve the fundamental problem of object segmentation using local and global image information with a geometric shape prior given by the model of PCA. To reach this objective, we have defined in Section 3.2 a shape-based functional to force the active contour to get a shape of interest whatever the position of the active contour in the image. Then in Section 3.3, we have proposed a Mumford and Shah-based functional to drive globally the shape model towards a homogeneous intensity region with the shape of interest. Experimental results have shown that our active contour is able to solve the problems of missing information and occlusion while being sensitive to local shape variations.

The shape model we used is the PCA model. As explained in Section 5, this model presents a good compromise between low complexity and acceptable shape priors. However, this model works well only if the probability density function (PDF) of the training set of the object of interest is Gaussian. If the true underlying PDF of the training set is not Gaussian (in presence of tumors in T1-WMR images for example) then more elaborated techniques such as non-parametric models are necessary.

Finally, note that the proposed model can capture only one object, which is a limitation since we loose the powerful property of the level set approach that can segment several objects simultaneously. A first solution to handle multiple objects would consist in associating structures by coupling the evolution equations. Other solutions would be to use the recent works of (Cremers et al. (2003, 2004)) and Tsai et al. (2004). Cremers et al. proposed in the context of variational level set methods a labeling function to indicate where to apply the shape prior in a given image. At last, Tsai et al. presented a parametric model to handle multiple shapes in a single mutual information-based cost criterion.

\section{Appendix: Existence of a Solution For our Minimization Problem}

This section deals with the mathematical study of

$$
\begin{aligned}
& \min _{\varphi, \mathbf{x}_{\mathrm{pca}}, \mathbf{x}_{\mathbf{T}}, u_{\text {in }}, u_{\text {out }}}\left\{F=\int_{\Omega}\left(\beta_{S} \hat{\phi}^{2}\left(x, \mathbf{x}_{\mathrm{pca}}, \mathbf{x}_{T}\right)\right.\right. \\
& \left.\left.+\beta_{b} g(x)\right)|\nabla H(\varphi)|+\beta_{r} F_{\text {region }}\left(\mathbf{x}_{\mathrm{pca}}, \mathbf{x}_{T}, u_{\text {in }}, u_{\text {out }}\right)\right\} .
\end{aligned}
$$

We follow the proofs of Chen et al. in Chen et al. (2002) and Vese and Chan (2001) to prove the existence of a minimizer for our proposed minimization problem using the direct method of the calculus of variations and compactness theorems on the space of functions with bounded variation.

The minimization problem is considered among characteristic functions $\chi_{E}$ of sets $E=\{x \in \Omega \mid \varphi(x) \geq$ $0\}$ with bounded variation. The vector of PCA eigencoefficients $\mathbf{x}_{\mathrm{pca}}=\left(\mathbf{x}_{\mathrm{pca}_{1}}, \ldots, \mathbf{x}_{\mathrm{pca}_{p}}\right)$ is defined on $\Omega_{\mathrm{pca}}=$ $\left[-3 \lambda_{1}, 3 \lambda_{1}\right] \times \cdots \times\left[-3 \lambda_{p}, 3 \lambda_{p}\right]$ and the vector of geometric transformations $\mathbf{x}_{T}=\left(s_{x}, s_{y}, \theta, s_{h}, T_{x}, T_{y}\right)$ is defined on $\Omega_{T}$. If $\Omega \subset \mathbf{R}^{2}$ is the domain of the original image $I$, say $\Omega=(0,255)^{2}$, then $\Omega_{T}=$ $(0,255]^{2} \times[-\pi, \pi) \times[-127,127] \times[-255,255]^{2}$. Functions $u_{\text {in }}$ and $u_{\text {out }}$ from Section 3.3 are supposed in $C^{1}(\Omega)$ since they are smoothed versions of the original image $u_{0}\left(u=u_{0}+\mu \Delta u\right.$ is the first order discretization of the linear heat diffusion equation $\partial_{t} u=\Delta u$ with $\left.u(0)=u_{0}\right)$.

We remind some definitions and theorems introduced in Evans and Gariepy (1992), Giusti (1985), Chen et al. (2002), Vese and Chan (2001) and Ambrosio (1989).

Definition 1. Let $\Omega \subset \mathbf{R}^{N}$ be an open set and let $f \in$ $L^{1}(\Omega)$. The total variation norm of $f$ is defined by

$$
T V(f)=\int_{\Omega}|\nabla f|=\sup _{\phi \in \Phi}\left\{\int_{\Omega} f(x) \operatorname{div} \phi(x)\right\},
$$

where $\Phi=\left\{\phi \in C_{0}^{1}\left(\Omega, \mathbf{R}^{N}\right)|| \phi(x) \mid \leq 1\right.$, on $\left.\Omega\right\}$.

Definition 2. A function $f \in L^{1}(\Omega)$ is said to have bounded variation in $\Omega$ if its distributional derivate satisfies $T V(f)<\infty$. We define $B V(\Omega)$ as the space of all functions in $L^{1}(\Omega)$ with bounded variation. The space $B V(\Omega)$ is a Banach space, endowed with 
the norm:

$$
\|f\|_{B V(\Omega)}=\|f\|_{L^{1}(\Omega)}+T V(f) .
$$

Theorem 1. A measurable subset $E$ of $\mathbf{R}^{N}$ has finite perimeter in $\Omega$ if and only if the characteristic function $\chi_{E} \in B V(\Omega)$. We have $\operatorname{per}_{\Omega}(E)=T V\left(\chi_{E}\right)=$ $\int_{\Omega}\left|\nabla \chi_{E}\right|<\infty$.

Definition 3. Let $\Omega \subset \mathbf{R}^{N}$ be an open set and let $f \in L^{1}(\Omega)$ and $\alpha(x)$ be positive valued continuous and bounded functions on $\Omega$. The weighted total variation norm of $f$ is defined by

$$
T V_{\alpha}(f)=\int_{\Omega} \alpha(x)|\nabla f|=\sup _{\phi \in \Phi_{\alpha}}\left\{\int_{\Omega} f(x) \operatorname{div} \phi(x)\right\},
$$

where

$$
\Phi_{\alpha}=\left\{\phi \in C_{0}^{1}\left(\Omega, \mathbf{R}^{N}\right)|| \phi(x) \mid \leq \alpha(x) \text {, on } \Omega\right\} .
$$

If a function $f$ has a finite weighted total variation norm in $\Omega$ then it also belongs to $B V(\Omega)$.

Definition 4. A function $f \in B V(\Omega)$ is a special function of bounded variation if its distributional derivative is given by

$$
|D f|=T V(f)+\int_{\Omega \cap S_{f}} J_{f} d \mathcal{H}^{N-1},
$$

where $J_{f}$ is the jump part defined on the set of points $S_{f}$ and $\mathcal{H}^{N-1}$ is the $(N-1)$-dimensional Hausdorff measure. The space of special functions of bounded variation $S B V(\Omega)$ is a Banach space, endowed with the norm:

$$
\|f\|_{S B V(\Omega)}=\|f\|_{L^{1}(\Omega)}+|D f| .
$$

Theorem 2. Let $\Omega \subset \mathbf{R}^{N}$ be an open set with a Lipschity boundary. If $\left\{f_{n}\right\}_{n \geq 1}$ is a bounded sequence in $B V(\Omega)$, then there exist a subsequence $\left\{f_{n_{j}}\right\}$ of $\left\{f_{n}\right\}$ and a function $f \in B V(\Omega)$, such that $f_{n_{j}} \rightarrow f$ strongly in $L^{p}(\Omega)$ for any $1 \leq p<N /(N-1)$ and

$$
T V(f) \leq \liminf _{n_{j} \rightarrow \infty} T V\left(f_{n_{j}}\right) .
$$

The following theorem is a generalization of the main theorem of Chen (2002).

Theorem 3. Let $\Omega \subset \mathbf{R}^{N}$ be an open set with a Lipschity boundary. If $\left\{f_{n}\right\}_{n \geq 1}$ is a bounded sequence in $B V(\Omega)$ and if $\left\{\alpha_{n}\right\}_{n \geq 1}$ is a sequence of positive valued continuous functions that uniformly converges to $\alpha$ on $\Omega$, then there exist subsequences $\left\{f_{n_{j}}\right\}$ of $\left\{f_{n}\right\}$ and $a$ function $f \in B V(\Omega)$ such that $f_{n_{j}} \rightarrow f$ strongly in $L^{p}(\Omega)$ for any $1 \leq p<N /(N-1)$ and

$$
T V_{\alpha}(f) \leq \liminf _{n_{j} \rightarrow \infty} T V_{\alpha_{n_{j}}}\left(f_{n_{j}}\right) .
$$

Theorem 4. Let $\Omega$ be a bounded and open subset of $\mathbf{R}^{2}$ and I be a given image with $I \in L^{\infty}(\Omega)$. The minimization problem (35) re-written in the following form

$$
\begin{aligned}
& \min _{\chi_{E}, \mathbf{x}_{\mathrm{pca}}, \mathbf{x}_{T}, u_{\mathrm{in}}, u_{\text {out }}}\left\{F=\int_{\Omega}\left(\beta_{s} \hat{\phi}^{2}\left(x, \mathbf{x}_{\mathrm{pca}}, \mathbf{x}_{T}\right)\right.\right. \\
& \left.+\beta_{b} g(x)\right)\left|\nabla \chi_{E}\right| \\
& \left.+\beta_{r} F_{\text {region }}\left(\mathbf{x}_{\mathrm{pca}}, \mathbf{x}_{T}, u_{\text {in }}, u_{\text {out }}\right)\right\}
\end{aligned}
$$

has a solution $\chi_{E} \in B V(\Omega), \mathbf{x}_{\mathrm{pca}} \in \Omega_{\mathrm{pca}}, \mathbf{x}_{T} \in \Omega_{T}$ and $u_{\text {in }}, u_{\text {out }} \in C^{1}(\Omega)$.

Proof: We use the direct method of the calculus of variations:

(A) Let $\left\{\chi_{E_{n}}, \mathbf{x}_{\mathrm{pca}_{n}}, \mathbf{x}_{T_{n}}, u_{\mathrm{in}_{n}}, u_{\mathrm{out}_{n}}\right\}_{n \geq 1}$ be a minimizing sequence of (45), i.e.

$$
\begin{aligned}
& \lim _{n \rightarrow \infty} F\left(\chi_{E}, \mathbf{x}_{\mathrm{pca}_{n}}, \mathbf{x}_{T_{n}}, u_{\mathrm{in}_{n}}, u_{\mathrm{out}_{n}}\right) \\
& \quad=\operatorname{\chi nf}_{E}, \mathbf{x}_{\mathrm{pca}}, \mathbf{x}_{T}, u_{\text {in }}, u_{\text {out }} \\
& F\left(\chi_{E}, \mathbf{x}_{\mathrm{pca}}, \mathbf{x}_{T}, u_{\text {in }}, u_{\text {out }}\right) .
\end{aligned}
$$

(B) Since $\chi_{E_{n}}$ is a sequence of characteristic functions of $E_{n}$, then $\chi_{E_{n}}(x) \in\{0,1\}$-a.e. in $\Omega$. A constant $M>0$ exists such that $\left\|\nabla \chi_{E_{n}}\right\|_{L^{1}(\Omega)} \leq M, \forall n \geq$ 1 . Therefore, $\chi_{E n}$ is a uniformly bounded sequence on $B V(\Omega)$.

Since $\left\{\mathbf{x}_{\mathrm{pca}_{n}}\right\}$ and $\left\{\mathbf{x}_{T_{n}}\right\}$ are bounded sequences on compact spaces $\Omega_{\text {pca }}$ and $\Omega_{T}$, subsequences that converge to limits $\mathbf{x}_{\mathrm{pca}}$ and $\mathbf{x}_{T}$ exist.

The integrand $f\left(x, \mathbf{x}_{\mathrm{pca}}, \mathbf{x}_{T}\right)=\beta_{s} \hat{\phi}^{2}+\beta_{b} g$ is positive and bounded because both functions $\hat{\phi}^{2}$ and 
$g$ are bounded on $\Omega$. Since the PCA is applied on continuous functions (SDFs) then the functions $\hat{\phi}$ and $f$ are also continuous and $f_{n}(\mathbf{x})=$ $f\left(\mathbf{x}, \mathbf{x}_{\mathrm{pca}_{n}}, \mathbf{x}_{T_{n}}\right)$ converges uniformly to $f$ on $\Omega$.

Following Theorem 3, a subsequence of $\chi_{E n}$ that converges to a function $\chi_{E}$ strongly in $L^{1}(\Omega)$ exists.

Moreover, Theorem 3 also states that

$$
\int_{\Omega} f\left|\nabla \chi_{E}\right| \leq \liminf \operatorname{nin}_{n_{j} \rightarrow \infty} \int_{\Omega} f_{n_{j}}\left|\nabla \chi_{E_{n j}}\right|
$$

(C) In the region-based functional defined in Eq. (24):

$$
\begin{aligned}
& F_{\text {region }}\left(\mathbf{x}_{\mathrm{pca}}, \mathbf{x}_{T}, u_{\mathrm{in}}, u_{\text {out }}\right) \\
& \quad=\int_{\Omega}\left(\Theta_{\text {in }} H\left(\hat{\phi}\left(\mathbf{x}_{\mathrm{pca}}, \mathbf{x}_{T}\right)\right)+\Theta_{\text {out }} H(-\hat{\phi})\right) d \Omega,
\end{aligned}
$$

the function $H\left(\hat{\phi}\left(\mathbf{x}_{\mathrm{pca}}, \mathbf{x}_{T}\right)\right)$ is a characteristic function $\chi_{G}$ of sets $G=\{x \in \Omega \mid \hat{\phi}(x) \geq 0\}$. So we have

$$
\begin{aligned}
& F_{\text {region }}\left(\mathbf{x}_{\text {pca }}, \mathbf{x}_{T}, u_{\text {in }}, u_{\text {out }}\right) \\
& \left.\quad=\int_{\Omega}\left(\Theta_{\text {in }} \chi_{G}\left(\mathbf{x}_{\text {pca }}, \mathbf{x}_{T}\right)\right)+\Theta_{\text {out }}\left(1-\chi_{G}\right)\right) d \Omega
\end{aligned}
$$

and we can define the function $u=u_{\text {in }} \chi_{G}+$ $u_{\text {out }}\left(1-\chi_{G}\right)$. The minimizing sequence of Eq. (45) implies

$$
\begin{aligned}
& \lim _{n \rightarrow \infty} F_{\text {region }}\left(\mathbf{x}_{\mathrm{pca}_{n}}, \mathbf{x}_{T_{n}}, u_{\text {in }_{n}}, u_{\text {out }_{n}}\right) \\
& \quad=\inf _{\mathbf{x}_{\text {pca }}, \mathbf{x}_{T}, u_{\text {in }}, u_{\text {out }}} F_{\text {region }}\left(\mathbf{x}_{\text {pca }}, \mathbf{x}_{T}, u_{\text {in }}, u_{\text {out }}\right)
\end{aligned}
$$

Since the function $\chi_{G}$ depends continuously on variables $\mathbf{x}_{\mathrm{pca}}$ and $\mathbf{x}_{T}$, we have $\chi_{G}\left(\mathbf{x}_{\mathrm{pca}_{n}}, \mathbf{x}_{T_{n}}\right)=\chi_{G_{n}}$ and $u_{n}=u_{\mathrm{in}_{n}} \chi_{G_{n}}+u_{\mathrm{out}_{n}}\left(1-\chi_{G_{n}}\right)$. According to Ambrosio's lemma (Ambrosio, 1989), we can deduce that there is a $u \in S B V(\Omega)$, such that a subsequence $u_{n_{j}}$ converges to $u$ a.e. in $B V-w *$ and

$$
\begin{aligned}
& F_{\text {region }}\left(\mathbf{x}_{\text {pca }}, \mathbf{x}_{T}, u_{\text {in }}, u_{\text {out }}\right) \\
& \quad=F_{\text {region }}(u) \leq \lim \inf _{n_{j} \rightarrow \infty} F_{\text {region }}\left(u_{n_{j}}\right),
\end{aligned}
$$

which means that $u$ is a minimizer of $F_{\text {region }}$. Then, by combining Eqs. (47) and (51), $\chi_{E}, \mathbf{x}_{\text {pca }}, \mathbf{x}_{T}, u_{\text {in }}$ and $u_{\text {out }}$ are minimizers of (45).

\section{Acknowledgments}

The authors would like to thank L. Jonasson and F. Mendels for their useful comments and R. M. Figueras i Ventura for providing us her genetic algorithm. Finally, they wish to thank all three unknown referees for their general and detailed comments, which improved very much the quality of the paper.

\section{References}

Adalsteinsson, D. and Sethian, J. 1995. A fast level set method for propagating interfaces. Journal of Computational Physics, 118:269-277.

Ambrosio, L. 1989. A compactness theorem for a new class of functions of bounded variation. Bolletino Della Unione Matematica Italiana, VII(4):857-881.

Biswal, B.B. and Hyde, J.S. 1997. Contour-based registration technique to differentiate between task-activated and head motioninduced signal variations in fMRI. Magnetic Resonance in Medecine, 38(3):470-476.

Bresson, X., Vandergheynst, P., and Thiran, J. 2004. A variational model for object segmentation using boundary information, statistical shape prior and the Mumford-Shah functional, ITS technical report 08.04, Signal Processing Institute, Swiss Federal Institute of Technology, Lausanne, Switzerland, http://itswww.epfl.ch.

Bresson, X., Vandergheynst, P., and Thiran, J.P. 2003. A priori information in image segmentation: Energy functional based on shape statistical model and image information. In IEEE International Conference on Image Processing, pp. 425-428.

Caselles, V., Kimmel, R., and Sapiro, G. 1997. Geodesic active contours. International Journal of Computer Vision, 22(1):61-79.

Chan, T.F. and Vese, L.A. 2001. Active contours without edges. IEEE Transactions on Image Processing, 10(2):266-277.

Charpiat, G., Faugeras, O., and Keriven, R. 2003. Shape metrics, warping and statistics. In IEEE International Conference on Image Processing, pp. 627-630.

Chen, Y., Tagare, H.D., Thiruvenkadam, S., Huang, F., Wilson, D., Gopinath, K.S., Briggsand, R.W., and Geiser, E.A. 2002. Using prior shapes in geometric active contours in a variational framework. International Journal of Computer Vision, 50(3):315-328.

Cootes, T. and Taylor, C. 1999. Statistical models of appearance for computer vision. Technical Report, University of Manchester.

Cremers, D., Kohlberger, T., and Schnörr, C. 2002. Nonlinear shape statistics in Mumford-Shah based segmentation. In European Conference on Computer Vision, pp. 93-108.

Cremers, D., Osher, S.J., and Soatto, S. 2004. Kernel density estimation and intrinsic alignment for knowledge-driven segmentation: Teaching level sets to walk. In Annual Pattern Recognition Symposium DAGM, Springer Verlag LNCS, vol. 3157, pp. 36-44.

Cremers, D., Sochen, N., and Schnörr, C. 2003. Towards recognitionbased variational segmentation using shape priors and dynamic labeling. In Scale-Space'03, LNCS 2695, pp. 388-400.

Cremers, D., Sochen, N., and Schnörr, C. 2004. Multiphase dynamic labeling for variational recognition-driven image segmentation. In European Conference on Computer Vision, pp. 74-86.

Cremers, D., Tischhäuser, F., Weickert, J., and Schnörr, C. 2002. Diffusion snakes: Introducing statistical shape knowledge into the 
Mumford-Shah functional. International Journal of Computer Vision, 50(3):295-313.

Davis, L. 1991. Handbook of Genetic Algorithms, Van Nostrand.

Evans, L.C. and Gariepy, R.F. 1992. Measure Theory and Fine Properties of Functions, Studies in advanced Mathematics, CRC Press.

Giusti, E. 1985. Minimal Surfaces and Functions of Bounded Variation. Birkhauser, Basel.

Jehan-Besson, S., Barlaud, M., and Aubert, G. 2003. DREAM2S: Deformable regions driven by an eulerian accurate minimization method for image and video segmentation. International Journal of Computer Vision, 53(1):45-70.

Jonasson, L., Bresson, X., Hagmann, P., Cuisenaire, O., Meuli, R., and Thiran, J.P. 2005. White matter fiber tract segmentation in DTMRI using geometric flows. Medical Image Analysis, 9(3):223236.

Kass, M., Witkin, A., and Terzopoulos, D. 1987. Snakes: active contour models. International Journal of Computer Vision, 321-331.

Kichenassamy, S., Kumar, A., Olver, P., Tannenbaum, A., and Yezzi, A.J. 1996. Conformal curvature flows: From phase transitions to active vision. In Archive for Rational Mechanics and Analysis, vol. 134, pp. 275-301.

Leventon, M. 2000. Statistical models for medical image analysis, Ph.D Thesis.

Leventon, M., Grimson, W., and Faugeras, O. 2000. Statistical shape influence in geodesic active contours. In IEEE International Conference of Computer Vision and Pattern Recognition, pp. 316-323.

Malladi, R., Kimmel, R., Adalsteinsson, D., Sapiro, G., Caselles, V., and Sethian, J.A. 1996. A geometric approach to segmentation and analysis of 3D medical images. In Mathematical Methods in Biomedical Image Analysis Workshop.

Numerical Recipies in $\mathrm{C}++$ and $C$, Cambridge University Press.

Mumford, D. and Shah, J. 1989. Optimal approximations of piecewise smooth functions and associated variational problems. Communications on Pure and Applied Mathematics, 42:577-685.

Osher, S. and Sethian, J.A. 1988. Fronts propagating with curvaturedependent speed: Algorithms based on Hamilton-Jacobi formulations. Journal of Computational Physics, 79(1):2-49.

Paragios, N. and Deriche, R. 2002. Geodesic active regions: A new framework to deal with frame partition problems in computer vision. Journal of Visual Communication and Image Representation, 13(1-2):249-268.

Paragios, N., Rousson, M., and Ramesh, V. 2003. Non-rigid registration using distance functions. Journal of Computer Vision and Image Understanding, 89(2-3):142-165.
Riklin-Raviv, T., Kiryati, N., and Sochen, N. 2004. Unlevel-sets: Geometry and prior-based segmentation. In European Conference on Computer Vision, pp. 50-61.

Rousson, M., Paragios, N., and Deriche, R. 2004. Implicit active shape models for 3D segmentation in MR imaging. In International Conference on Medical Image Computing and Computer Assisted Intervention (MICCAI), pp. 209216.

Samson, C., Blanc-Féraud, L., Aubert, G., and Zerubia, J. 2000. A level set model for image classification. International Journal of Computer Vision, 40(3):187-197.

Tsai, A., Wells, W., Tempany, C., Grimson, E., and Willsky, A. 2004. Mutual information in coupled multi-shape model for medical image segmentation. Medical Image Analysis, 8(4):429445.

Tsai, A., Yezzi, A., Wells, W., Tempany, C., Tucker, D., Fan, A., Grimson, W.E., and Willsky, A. 2001. Model-based curve evolution techniques for image segmentation. In IEEE International Conference of Computer Vision and Pattern Recognition, pp. 463468.

Tsai, A., Yezzi, A., Wells, W., Tempany, C., Tucker, D., Fan, A., Grimson, W.E. and Willsky, A. 2003. A shape-based approach to the segmentation of medical imagery using level sets. IEEE Transactions on Medical Imaging, 22(2):137154.

Unser, M. 1999. Splines: A perfect fit for signal and image processing. IEEE Signal Processing Magazine, 16(6):22-38.

Vese, L. and Chan, T. 2001. A multiphase level set framework for image segmentation using the Mumford and Shah model, UCLA CAM Report 01-25.

Vese, L.A. and Chan, T.F. 2002. A multiphase level set framework for image segmentation using the Mumford and Shah model. International Journal of Computer Vision, 50(3):271293.

Yezzi Jr., A., Kichenassamy, S., Kumar, A., Olver, P.J., and Tannenbaum, A. 1997. A geometric snake model for segmentation of medical imagery. IEEE Transactions on Medical Imaging, 16(2):199209.

Yezzi, A., Tsai, A., and Willsky, A. 1999. A statistical approach to snakes for bimodal and trimodal imagery. In International Conference of Computer Vision, pp. 898-903.

Zhao, H., Chan, T., Merriman, B., and Osher, S. 1996. A variational level set approach to multiphase motion. Journal of Computational Physics, 127:179-195. 